\title{
Price Dispersion, Private Uncertainty, and Endogenous Nominal Rigidities
}

\author{
Gaetano Gaballo ${ }^{1}$
}

\author{
December 2017, WP \#653
}

\begin{abstract}
This paper shows that when agents learn from prices, large private uncertainty may result from a small amount of heterogeneity. As in a Phelps-Lucas island model, final producers look at the prices of their local inputs to infer aggregate conditions. However, market linkages between islands make the informativeness of local prices endogenous to general equilibrium relations. In this context, I show that a vanishingly small heterogeneity in local conditions is enough to generate an equilibrium in which prices are rigid to aggregate shocks and transmit only partial information. I use this insight as a microfoundation for price rigidity in an otherwise frictionless monetary model and show that even a tiny amount of dispersion in fundamentals can lead to large non-neutrality of money. ${ }^{2}$
\end{abstract}

Keywords: learning from prices, expectational coordination, dispersed information.

JEL classification: D82, D83, E3

1 Banque de France, gaetano.gaballo@banque-france.fr

2 This paper partly incorporates results from "Endogenous Signals and Multiplicity". I would like to thank George Evans, Roger Guesnerie, Christian Hellwig, David K. Levine, Ramon Marimon and Michael Woodford for their support at different stages of this project. I am grateful to Sushant Acharya, George Marios Angeletos, Robert Barsky, Jess Benhabib, Ryan Chahrour, Gabriel Desgranges, Alexandre Kohlhas, Guillermo Ordoñez, Robert Ulbricht, François Velde, Pierre-Olivier Weill for insightful comments, and the editor Dimitri Vayanos and three anonymous referees for their critical remarks, which greatly helped to improve the quality of this paper. It goes without saying that any errors are my own.

Working Papers reflect the opinions of the authors and do not necessarily express the views of the Banque de France. This document is available on publications.banque-france.fr/en 


\section{NON-TECHNICAL SUMMARY}

Aggregate price rigidity is an important regularity of business cycles. In particular, the effectiveness of monetary policy relies on the sluggish response of prices to aggregate shocks. Therefore, what determines aggregate price rigidity is one of the crucial questions in Macroeconomics. Robert Lucas Jr. and Edmund Phelps have been the first arguing that delays in the acquisition of information about a change in aggregate conditions may generate aggregate price rigidity. To formalize this idea they created the fiction of an economy segmented in islands. Each producer only sees the local prices of the inputs traded in his own island; for this reason, she gets confused on whether price changes reflect local rather than global fluctuations. Although very influential, one problem with these models is that markets need to be severely fragmented and economic fundamentals be widely dispersed for the model to predict the high level of price rigidity that we observe in data.

This paper demonstrates that the Lucas-Phelps insight is much more robust than before thought. I show that when agents learn from prices, arbitrarily small dispersion in fundamentals may lead to substantial price rigidity. The result is obtained in a typical island economy by allowing for market linkages across islands. In particular, I model local inputs being in turn the output of intermediate local firms competing on a global market for the same common factor. The price of this global factor determines each local price of inputs jointly with other local disturbances. Thus, how much prices respond to global, rather than local, factors is determined by market forces. As a result, the information revealed by local prices about aggregate conditions can remain very small, even in the limit in which market fundamentals are nearly homogeneous across islands and producers all observe almost the same price.

The figure below illustrates how such equilibria may form in a simple demand-supply graph in the market of local inputs. In the panels below we measure prices and quantities on the $\mathrm{x}$-axis and $\mathrm{y}$-axis respectively; moreover, the grey-horizontal line $\mathrm{k}$ denotes a supply schedule, whereas the black-steep line D a demand schedule. Suppose that both demand and supply move upwards in response to information about a positive aggregate shock, namely theta. Consider now that an aggregate shock is known by sellers which shifts supply upwards (dotted gray arrow). In panel (a) I represent a benchmark case in which demand does not move as buyers have no information about theta: in such a case the price of equilibrium $r$ becomes negative for the market to clear a larger supply. In panel (b) I illustrate the case when buyers have full information: demand shifts upward (solid black arrow) and the equilibrium price moves right. In particular when demand moves more than supply than the equilibrium price becomes positive. Under these conditions, an equilibrium as the one plotted in panel (c) may emerge. In such equilibrium, buyers only receive partial information about theta so that demand raises just enough to makes prices remain arbitrarily close to steady state. In this configuration the reaction of prices to the aggregate shock may be so small that any idiosyncratic noise - no matter how small - may make buyers partly informed and so fulfill the equilibrium.

In the paper, I first present a stylized model to discuss the main mechanism. Then I introduce a more standard macro-model to show that the same mechanism can have 
modern DSGE microfoundations and that previous insights carry over into richer settings.

These results shed new light on the nature of price rigidities. Previous work typically relies on frictions in the availability or use of information about input costs to explain why producers do not readily adjust prices. Calvo pricing (Calvo, 1983), menu costs (Sheshinski and Yoram, 1977), inattentiveness (Reis, 2006) or rational inattention (Wienderholt and Mackowiak, 2009) are a few popular examples of an ever-growing literature. In my model, in contrast, producers perceive their marginal costs precisely and are not constrained in their price setting.

\section{The Effects of Information on Equilibrium Prices}

(a)

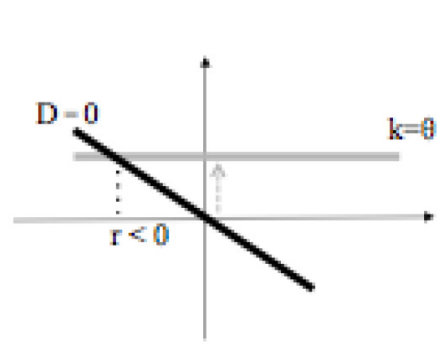

(b)

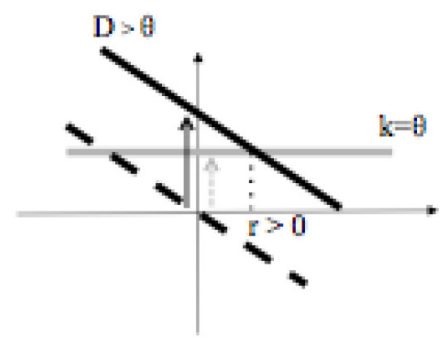

(c)

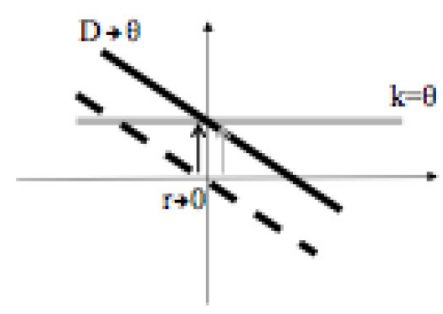

Figure 1: The effect of a positive $\theta$ on average supply (light gray) and average dernand (dark gray) in cupital markets. Arrows illustrate the shifts of the two curves in three cases: no information (panel a), perfect information (panel b) and partial information (panel c). Solid (resp. dashed) arrows denote shifts that (resp. do not) depend on expectations. In panel (b) and (c) the thick dashed line represents the no-information demand.

\section{Dispersion des prix, incertitude privée et rigidités nominales endogènes}

Cet article montre que lorsque les agents apprennent à partir des prix, une grande incertitude privée peut résulter d'une faible hétérogénéité. Comme dans le modèle des îles de Phelps et Lucas, les producteurs finaux examinent les prix de leurs facteurs productifs locaux pour former des anticipations des conditions agrégées. Cependant, les liens de marché entre les îles rendent l'informativité des prix locaux endogène aux relations d'équilibre général. Dans ce contexte, je montre qu'une hétérogénéité des conditions locales infinitésimale est suffisante pour générer un équilibre dans lequel les prix sont rigides aux chocs agrégés et ne transmettent que de l'information partielle. J'utilise cette idée comme un fondement microéconomique de la rigidité des prix dans un modèle monétaire sans aucune autre friction et montre que même une infime quantité de dispersion dans les fondamentaux peut conduire à une grande non-neutralité de chocs monétaires.

Mots-clés : apprentissage des prix, coordination des anticipations, information dispersée.

Les Documents de travail reflètent les idées personnelles de leurs auteurs et n'expriment pas nécessairement la position de la Banque de France. Ce document est disponible sur publications.banque-france.fr 
"The mere fact that there is one price for any commodity - or rather that local prices are connected in a manner determined by the cost of transport, etc. - brings about the solution which (it is just conceptually possible) might have been arrived at by one single mind possessing all the information which is in fact dispersed among all the people involved in the process." Hayek (1945)

\section{Introduction}

The idea that private uncertainty can be a major cause of aggregate price rigidity has a noble tradition dating back to Phelps (1970) and Lucas (1972, 1973, 1975). Most of the subsequent literature has built upon their insights by assuming that agents privately observe exogenously-specified signals. This simplification, although useful in many contexts, has led to criticism about the lack of a clear empirical counterpart for these informational frictions.

A natural response is to think about private signals as the prices that agents observe in the local markets in which they trade. However, this interpretation has often been thought to put inescapable limits on the market structure that can be considered. In particular, markets need to be severely fragmented to prevent prices from aggregating information and dissipating uncertainty as Hayek (1945) argued. In addition, prices need to be widely dispersed across markets to let private uncertainty play a major role. These difficulties have cast doubts on the generality and realism of incomplete information models.

This paper contradicts such skepticism, showing that, when agents learn from prices, externalities in the aggregation of information can generate large private uncertainty no matter how small the heterogeneity in the economy. To show this, I extend the typical Phelps-Lucas setting - in which producers learn from the prices on their own island - by introducing market linkages between islands. This innovation makes the informativeness of local prices endogenous to general equilibrium. As a result, the information revealed by local prices about aggregate conditions can remain very small, even in the limit in which market fundamentals are nearly homogeneous across islands and producers all observe almost the same price.

The key mechanism presented in this paper relies neither on price-setting nor on the presence of money, although workhorse monetary models constitute a natural application. To highlight this generality, I present two models: one real and one monetary.

The first model is a stylized real economy in which competitive producers - who are located on islands - acquire local capital before observing aggregate productivity. Each variety of local capital is produced by intermediate firms that compete for the same endowment across islands. The price of this global factor determines each local price of capital jointly with other local disturbances. Thus, how much prices respond to global, rather than local, factors is determined by market forces.

In the absence of any heterogeneity, local prices comove perfectly with aggregate productivity. In this case, producers are perfectly informed by the prices of their local 
inputs and they fulfill the social optimum as a unique equilibrium outcome.

Nevertheless, with a vanishingly small heterogeneity in intermediate production, I prove that two types of equilibrium exist. One equilibrium inherits by continuity all the good properties of the perfect-information equilibrium. The other, which I tag dispersed information limit equilibrium, exhibits features in stark contrast: local prices are unresponsive to the aggregate shock, and there is a large misallocation of capital across islands. A dispersed-information limit can be understood as the combination of two externalities that emerge in the use of information. ${ }^{1}$

First, as producers react more to prices, prices must move less with productivity. This is because when prices cause large shifts in expectations, smaller price adjustments are enough to induce market clearing. However, as prices move less with productivity, a producer should react more to prices, as a small change in price means a large revision in productivity. This feedback entails a strong complementarity: as producers' reactions increase, it is optimal for a single producer to react even more.

Second, when producers' reaction is so strong that the response of local prices to productivity shrinks to a sufficiently small number, small local disturbances affect producers' inference; that is, local prices lose precision. This feedback entails a substitutability: as producers' reactions increase, it is optimal for a single producer to react less.

A dispersed-information equilibrium obtains when substitutability balances complementarity so that the optimal reaction is equal to the average reaction. Thus, no matter how small local disturbances are, there is an equilibrium in which producers react strongly to prices, prices move little and producers are privately uncertain. However, such an equilibrium cannot emerge without local disturbances, because otherwise the substitutability mechanism does not kick in.

I also study the issue of equilibrium stability. I show that in a perfect-information limit equilibrium, a small doubt about how much others will react leads producers to consider large deviations from the equilibrium, which makes it hard for them to coordinate their actions. This is not the case in a dispersed information limit, which instead exhibits contracting properties in higher-order beliefs.

The aim of the second model is to show that a dispersed-information limit equilibrium can have standard DSGE microfoundations and that previous insights carry over into richer settings. In the extended economy, producers acquire local capital knowing about productivity, but are now uncertain about the average price level. In particular, I take the usual DSGE structure with a representative household and monopolistically competitive price setters that hire capital and labor. Capital is produced locally by using a commonly priced factor, exactly as in the previous economy. Wages are also local, but correlate with a money shock that shifts the labor supply in all islands. As producers do not observe money shocks, they look at the prices of their inputs to predict inflation, as in a Phelps-

\footnotetext{
${ }^{1}$ Angeletos and Pavan (2007) study the use of information in models with convex payoffs and signals with exogenous precision. In contrast, here the use of information affects the precision of the price signals, which are endogenous to equilibrium relations.
} 
Lucas model.

The capital market exhibits the same mechanism as in the stylized model. Without any heterogeneity, this economy has a unique equilibrium in which producers can perfectly infer changes in money supply from the local price of capital; as a result, money shocks are neutral. However, even small differences across islands can produce a dispersedinformation limit equilibrium. In this equilibrium, money shocks have real effects as prices become endogenously rigid to aggregate conditions and local shocks produce a wide cross-section of beliefs that magnifies heterogeneity in final allocation.

The existence of dispersed-information limit equilibria sheds new light on the nature of price rigidities. Previous work typically relies on frictions in the availability or use of information about input costs to explain why producers do not readily adjust prices. Calvo pricing (Calvo, 1983), menu costs (Sheshinski and Weiss, 1977), inattentiveness (Reis, 2006) or rational inattention (Mackowiak and Wiederholt, 2009) are a few popular examples of an ever-growing literature. Here, in contrast, producers perceive their marginal costs precisely and are not constrained in their price setting.

Angeletos and La'O (2010) provide an insightful analysis of the effects of imperfect information over the business cycle, although their information structure is not microfounded and the precision of the signals is exogenous. Amador and Weill (2010) present a fully microfounded model to study the welfare consequences of learning from prices. They also find the possibility of a multiplicity that, unlike that characterized in this paper, vanishes with small dispersion or low enough pay-off complementarities. ${ }^{2}$

The relationship between private uncertainty, prices and multiplicity has been discussed in the global games literature. Morris and Shin (1998) demonstrate that arbitrarily small private uncertainty about fundamentals leads to a unique equilibrium in an otherwise multiple-equilibrium model; Angeletos and Werning (2006), and Hellwig et al. (2006) clarify that this result holds in the absence of sufficiently informative public prices. In contrast, this paper demonstrates that an arbitrarily small price dispersion can cause multiplicity in an otherwise unique-equilibrium model.

Benhabib et al. (2015) show that partly revealing equilibria - called sentiments - can coexist with a fully revealing equilibrium because of the endogenous nature of information. Nonetheless, their multiplicity collapses on the perfect-information outcome in the limit of no dispersion. Chahrour and Gaballo (2017) show that sentiment equilibria can be characterized as dispersed-information limit equilibria pushing the variance of a common - rather than an idiosyncratic - informational shock to the limit of zero.

In the game-theoretic language of Bergemann and Morris (2013), dispersed informa-

\footnotetext{
${ }^{2}$ Many authors have found the possibility of multiple REE due to imperfect information with endogenous precision (not necessarily learning from prices). They either restrict the coefficient region to focus on a unique equilibrium or characterize a multiplicity that vanishes with small enough dispersion of signals. A non-exhaustive list includes: Angeletos et al. (2010), Angeletos and La'O (2008), Ganguli and Yang (2009), Manzano and Vives (2011), Vives (2017) and Desgranges and Rochon (2013). In the CARA asset pricing literature, numerous examples of multiple noisy REE exist that rely on the presence of risk aversion to the conditional variance of asset returns (see Walker and Whiteman (2007)).
} 
tion equilibria are Bayes Nash equilibria, i.e., Bayes correlated equilibria decentralized by a particular information structure. Bergemann et al. (2015) explore the stochastic properties of the set of Bayes correlated equilibria by means of signals with exogenous precision. This paper contributes to that agenda by showing how Bayes correlated equilibria can be decentralized via an endogenous information structure that is microfounded within a system of competitive prices.

\section{A simple real economy}

This section provides a simple model to illustrate the core results of the paper.

I present a real economy in which final producers look at the competitive price of their local input to infer aggregate productivity. I demonstrate the existence of equilibria in which, no matter how small local differences are, prices are imperfect signals of productivity. As a result, a tiny heterogeneity in fundamentals can lead to large welfare losses, although the unconstrained first best is still a market outcome. I also demonstrate that out-of-equilibrium dynamics select the suboptimal equilibria.

\subsection{A model of decentralized cross-sectional allocation}

Consider an economy constituted by a continuum of islands indexed by $i \in(0,1)$. There are different types of goods: (i) an endowment $Z>0$ of homogeneous raw capital, (ii) a local capital, one type for each island, and (iii) an homogeneous consumption good. Raw capital can produce any variety of local capital; any variety of local capital can produce consumption.

Each island is inhabited by atomistic agents who have utility simply equal to their consumption. Agents are of two types - intermediate and final producers - and are evenly distributed across the islands. Intermediate producers transform raw capital into local capital; final producers transform local capital into consumption. Raw capital is owned in equal shares by intermediate producers, but can be traded between islands. Production is stochastic and partially correlated across islands and types.

The timing is as follows. In the first stage, all shocks realize. In the second stage, the markets for raw capital and all local capital open and close simultaneously; all prices in these markets are non-contingent claims on final production. In the third stage, the consumption good is produced and allocated according to prices; final producers are residual claimants of their own production. ${ }^{3}$

We assume that productivity can be observed only once production has been implemented. Therefore, final producers acquire inputs without knowing the actual quantity that they will produce, whereas intermediate producers observe their productivity when

\footnotetext{
${ }^{3}$ We shall assume that producers have a sufficiently large endowment in consumption to allow for potentially negative residual claims. Also note that the linear specification of the utility function makes the absence of a market for consumption irrelevant.
} 
they supply local capital. As productivity shocks are correlated, local market prices provide final producers with useful information.

Formally, a final producer on island $i$ buys a quantity of local capital $K_{i}$ to maximize her expected consumption; that is, she solves

$$
\max _{K_{i}}\left\{\mathrm{E}\left[Y_{(i)} \mid R_{i}\right]-R_{i} K_{i}\right\}
$$

where $\mathrm{E}\left[Y_{(i)} \mid R_{i}\right]$ is the expectation of her own production $Y_{(i)}$ conditional on the realization of $R_{i}{ }^{4}$ In fact, final producers forecast productivity using the price of their own local input as a predictor. Their technology,

$$
Y_{(i)} \equiv \mathrm{e}^{\mu \theta} K_{i}^{\alpha}
$$

exhibits decreasing returns to scale measured by $\alpha \in(0,1)$, and it is hit by a normally distributed aggregate productivity shock $\theta \sim \mathcal{N}(0,1)$ with intensity $\mu>0 .^{5}$

An intermediate producer on island $i$ buys a quantity of raw capital $Z_{(i)}$ to maximize her own profits; that is, she solves

$$
\max _{Z_{(i)}}\left\{R_{i} K_{i}^{s}-Q Z_{(i)}\right\}
$$

where $R_{i}$ is the local price of capital type $i$ and $Q$ is the global price of raw capital. Their technology, ${ }^{6}$

$$
K_{i}^{s} \equiv \mathrm{e}^{\theta+\eta_{i}} Z_{(i)},
$$

is subject to the same aggregate shock augmented by an independently distributed local productivity shock $\eta_{i} \sim \mathcal{N}\left(0, \sigma^{2}\right)$, which accounts for variations in local conditions.

Finally, given that total production $Y \equiv \int Y_{(i)}$ di is consumed and utility is linear in consumption, $Y$ also represents a utilitarian measure of social welfare.

\subsection{Learning from prices}

\section{Definition of an equilibrium}

I restrict the analysis to equilibria with a log-normal representation, so that expectations about $\theta$ have a linear characterization, as usually assumed in the literature on noisy rational expectations from Grossman (1976) and Hellwig (1980) onward. A formal definition of an equilibrium follows.

\footnotetext{
${ }^{4}$ Hereafter, subscripts in parentheses denote the quantity of a homogeneous good acquired on an island, whereas simple subscripts are used for the differentiated good produced on an island.

${ }^{5} \mathcal{N}($ m, var $)$ conventionally denotes a Normal distribution with mean $m$ and variance var. The variance of the aggregate shock is normalized for convenience of notation. None of the results of this paper hinge on this normalization; considering a different value for the variance of aggregate shocks requires multiplying the variance of idiosyncratic shocks for that same value, which leaves their ratio $\sigma^{2}$ unaffected.

${ }^{6}$ In Appendix A.6 I discuss the case with decreasing returns to scale. Decreasing returns restrict the conditions for the existence of dispersed-information limit equilibria; this feature emerges here due to the specific microfoundations of this simple model. See footnote 16 .
} 
Definition 1. A rational expectation equilibrium is a collection of prices $\left(Q,\left\{R_{i}\right\}_{i \in[0,1]}\right)$, quantities $\left\{Y_{(i)}, K_{i}, K_{i}^{s}, Z_{(i)}\right\}_{i \in[0,1]}$ and island-specific expectations $\left\{\mathrm{E}\left[Y_{(i)} \mid R_{i}\right]\right\}_{i \in[0,1]}$, contingent on the stochastic realizations $\left(\theta,\left\{\eta_{i}\right\}_{i \in[0,1]}\right)$, such that:

- (optimality) agents optimize their actions according to the prices they observe;

- (market clearing) all markets clear, i.e., $\int Z_{(i)} \mathrm{di}=Z, K_{i}^{s}=K_{i}$ in each $i \in(0,1)$;

- (log-normality) prices and quantities are log-normally distributed.

The first-order conditions of the two types are

$$
\begin{aligned}
R_{i} e^{\theta+\eta_{i}} & =Q, \\
\alpha \mathrm{E}\left[e^{\mu \theta} \mid R_{i}\right] K_{i}^{-(1-\alpha)} & =R_{i} .
\end{aligned}
$$

In a log-normal equilibrium, $R_{i}$ can be expressed as $R_{i}=\bar{R}_{i} e^{r_{i}}$, where $\bar{R}_{i}$ represents the median realization of $R_{i}$ and $r_{i} \equiv \log R_{i}-\log \bar{R}_{i}$ its stochastic component, which is a linear combination of $\theta$ and $\eta_{i}$. Analogously, we can define: $Q=\bar{Q} e^{q}, K_{i}=$ $\bar{K}_{i} e^{k_{i}}, Z_{(i)}=\bar{Z}_{(i)} e^{z_{(i)}}$. Log-normality also applies to expectations, so that $\mathrm{E}\left[e^{\mu \theta} \mid R_{i}\right]=$ $e^{\mu \mathrm{E}\left[\theta \mid r_{i}\right]+\mu^{2} \operatorname{var}\left(\theta \mid r_{i}\right) / 2}$ where $\operatorname{var}\left(\theta \mid r_{i}\right)$ is the variance of $\theta$ conditional on $r_{i}$.

All the relations above must hold state by state. In particular, for $\left(\theta, \eta_{i}\right)=(0,0)$, we have $\bar{K}_{i}^{s}=\bar{Z}_{(i)}, \bar{R}_{i}=\bar{Q}$ and $\alpha e^{\mu^{2} \operatorname{var}\left(\theta \mid r_{i}\right) / 2} \bar{K}_{i}^{-(1-\alpha)}=\bar{R}_{i}$. We can use these to exactly transform (4), (5) and (6) into a log-linear system:

$$
\begin{aligned}
k_{i}^{s} & =\theta+\eta_{i}+z_{(i)}, \\
r_{i}+\theta+\eta_{i} & =q, \\
\mu \mathrm{E}\left[\theta \mid r_{i}\right]-(1-\alpha) k_{i} & =r_{i},
\end{aligned}
$$

which, together with $\int z_{(i)} \mathrm{di}=0$, identifies an equilibrium. Before solving the model, it is useful to discuss the key mechanics embedded in local capital markets.

\section{Inspecting capital markets}

Figure 1 illustrates how local prices depend on final producers' knowledge about productivity. The average price $r \equiv \int r_{i}$ di and quantity $k \equiv \int k_{i}$ di are measured on the $\mathrm{x}$-axis and $\mathrm{y}$-axis respectively. I consider the case of a given realization $\theta>0$ with $\mu>1-\alpha$. The downward-sloping line represents demand

$$
k=\underbrace{\frac{\mu}{1-\alpha} \int \mathrm{E}\left[\theta \mid r_{i}\right] \mathrm{di}}_{\equiv D}-\frac{1}{1-\alpha} r,
$$

which is obtained by averaging over (9). The horizontal line denotes the supply, $k=\theta$, which obtains using $k_{i}=k_{i}^{s}$ and $\int z_{(i)} \mathrm{di}=0$ into (7). The intersection of demand and supply determines the equilibrium $r$ for a given $\theta$. Note that producers' expectations act 
(a)

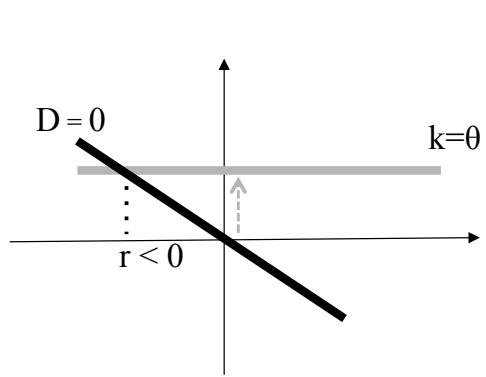

(b)

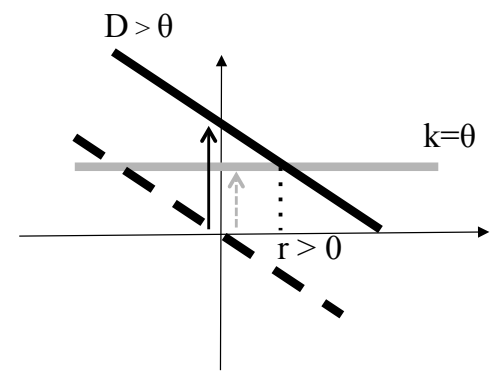

(c)

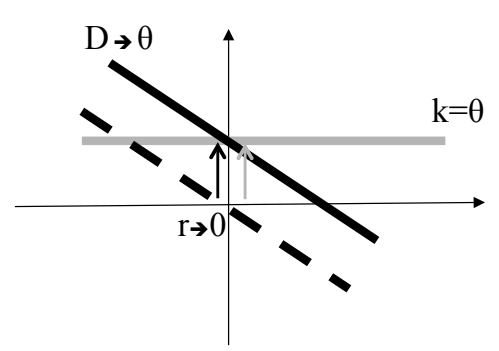

Figure 1: The effect of a positive $\theta$ on average supply (light gray) and average demand (dark gray) in capital markets. Arrows illustrate the shifts of the two curves in three cases: no information (panel a), perfect information (panel b) and partial information (panel c). Solid (resp. dashed) arrows denote shifts that (resp. do not) depend on expectations. In panel (b) and (c) the thick dashed line represents the no-information demand.

as a demand shifter: the higher the expected $\theta$, the higher the demand and, in turn, the higher the equilibrium $r$.

Panel (a) plots the no-information case with $\mathrm{E}\left[\theta \mid r_{i}\right]=0$ for which $r=-(1-\alpha) \theta$. In the absence of any information, a greater supply induces a lower price due to decreasing returns. In this case, $\theta$ and $r$ are negatively correlated.

Panel (b) plots the perfect-information case with $\mathrm{E}\left[\theta \mid r_{i}\right]=\theta$ for which $r=(\mu-(1-$ $\alpha)) \theta$. Despite a larger supply, producers ask for more because they know that productivity is high. In particular, when $\mu>1-\alpha$, the loss in returns is more than compensated for by higher productivity. In this case, demand moves more than supply and $\theta$ and $r$ are now positively correlated.

The condition $\mu>1-\alpha$ means that prices are more sensitive to demand (producers' appetite) rather than to supply (quantity on the market). Only in this case, producers' information makes a difference in the correlation between $r$ and $\theta$ : it is negative in the case of no information, and positive in case of perfect information. By continuity, this implies that there must be a degree of uncertainty such that the upward shift in demand just offsets the downward shift in supply, so that the equilibrium $r$ tends not to move. This situation is plotted in panel (c), where the average expectation is now given by $\int \mathrm{E}\left[\theta \mid r_{i}\right] \mathrm{di}=(1-\alpha) \theta / \mu$.

The last scenario is one in which final producers are only partially informed about $\theta$ and, as a consequence, the average price $r$ reacts little to $\theta$. This situation can be an equilibrium in our economy where producers learn from prices. To see this intuitively, use (9) to express the price that producers observe as a private noisy signal of $r$; that is, $r_{i}=r-\eta_{i}$. When $r$ fluctuates a little, even small local shocks are enough to blur producers' inference.

In what follows, I formally characterize such equilibrium in terms of producers' re- 
action to prices. I will show that, although, without heterogeneity, only the perfect information equilibrium exists, in the limit of no heterogeneity, a dispersed-information equilibrium also exists that entails the configuration shown in panel (c).

\section{Dispersed-information limit}

The first step in solving the signal extraction problem is to express $r_{i}$ in a convenient way. We can integrate (8) and (9) to establish

$$
r=q-\theta=\int\left(\mu \mathrm{E}\left[\theta \mid r_{i}\right]-(1-\alpha) k_{i}\right) \text { di. }
$$

Then, using (11) to substitute $q-\theta$ into (8), we obtain the local market clearing price as

$$
r_{i}=\mu \int \mathrm{E}\left[\theta \mid r_{i}\right] \mathrm{di}-(1-\alpha) \theta-\eta_{i}
$$

where we used the fact that $\int k_{i} \mathrm{di}=\theta$. From the point of view of the final producer in island $i$, the local price of her input constitutes a private signal of an underlying aggregate endogenous state. The presence of private noise $\eta_{i}$ generates confusion about the nature - local or global - of price fluctuations. ${ }^{7}$

To find the set of equilibria, one needs to solve the fixed point problem, which is implicit in (12), and write down the profile of final producers' expectations as a function of shocks. There are many ways to tackle this problem; I will choose the one that provides a well-defined out-of-equilibrium characterization.

Let us start from the observation that when random variables are normally distributed, the optimal forecasting rule is linear in the realization of the signal. Hence, agent $i$ 's forecast is written as $\mathrm{E}\left[\theta \mid r_{i}\right]=b_{i} r_{i}$; that is,

$$
\mathrm{E}\left[\theta \mid r_{i}\right]=b_{i}\left(\mu \int \mathrm{E}\left[\theta \mid r_{i}\right] \mathrm{di}-(1-\alpha) \theta-\eta_{i}\right)
$$

where $b_{i}$ is a deterministic weight that measures the reaction of expectations type $i$ to the price signal $r_{i}$. Given that all agents use the above rule, then by definition the aggregate expectation is

$$
\int \mathrm{E}\left[\theta \mid r_{i}\right] \mathrm{di}=-\frac{b(1-\alpha)}{1-b \mu} \theta
$$

provided by $b \neq 1$, where $b \equiv \int b_{i}$ di denotes the average reaction across agents. ${ }^{8}$ Therefore, the price signal can be rewritten as

$$
r_{i}=-\frac{1-\alpha}{1-\mu b} \theta-\eta_{i}
$$

\footnotetext{
${ }^{7}$ In contrast to the popular framework introduced by Grossman (1976), the input price also responds to the actual fundamental realization when all producers are uninformed. This is because the price embodies information from the intermediate producers, who actually observe the realization at the time of trade. This avoids the problem of the implementability of the perfect information equilibrium which is often discussed in the literature.

${ }^{8} \mathrm{I}$ implicitly rule out cases in which $\int b_{i} \eta_{i} \mathrm{di} \neq 0$. Note that such cases violate the linearity of the forecasting rule.
} 
implying that as $b$ becomes large in absolute value, price volatility declines.

This property crucially depends on market clearing: as expectations become more sensitive to prices, prices have to move less to clear the market. In particular, the price becomes less responsive to the aggregate shock, whereas the impact of local disturbances remains unchanged. This implies that the precision of the price signal

$$
\tau(b)=\frac{(1-\alpha)^{2}}{(1-b \mu)^{2} \sigma^{2}}
$$

decreases for $b$ that is sufficiently large in absolute value. ${ }^{9}$

Notice that relations (13)-(16) hold for any given profile of individual reactions $\left\{b_{i}\right\}_{i \in[0,1]}$. However, expectations comply with Bayesian updating only when each weight $b_{i}$ satisfies the orthogonality condition $\mathrm{E}\left[r_{i}\left(\theta-b_{i} r_{i}\right)\right]=0$. Hence, a REE is characterized by a symmetric profile of weights $b_{i}(b)=b$ for each $i \in(0,1)$. The popular OLS formula gives the individual best reaction function ${ }^{10}$

$$
b_{i}(b)=\frac{\operatorname{cov}\left(\theta, r_{i}\right)}{\operatorname{var}\left(r_{i}\right)}=\underbrace{\frac{\mu b-1}{1-\alpha}}_{\text {scale }} \underbrace{\frac{\tau(b)}{1+\tau(b)}}_{\text {precision }},
$$

as composed of a scale factor and a precision factor. Changes in $b$ affect both factors in opposite ways. The scale factor exhibits complementarity, whereas the precision factor exhibits substitutability. On the one hand, a larger collective reaction $b$ implies a larger scale factor, as the same changes in prices map into larger variations in productivity. On the other hand, an absolute larger collective reaction $b$ implies a lower precision factor as prices becomes less informative. These two forces give $b_{i}(b)$ a typical S-shape that gets more pronounced as $\sigma$ shrinks. This is because a smaller $\sigma$ requires an absolute larger $b$ to let the precision factor have a bite. The nonlinearity of $b_{i}(b)$ generates a multiplicity of equilibria, as stated by the following proposition.

Proposition 1. In the perfect-information case $\sigma^{2}=0$ a unique equilibrium exists, which is characterized by $b_{*}=(\mu-(1-\alpha))^{-1}$. For $\sigma^{2} \rightarrow 0$, if and only if $\mu>1-\alpha$, three equilibria exist: a perfect-information limit equilibrium, characterized by $a b_{\circ} \rightarrow$ $b_{*}$, and two dispersed-information limit equilibria, characterized by $b_{+}>0$ and $b_{-}<0$ respectively, such that $\left|b_{ \pm}\right|>M$ where $M$ is an arbitrarily large finite value; otherwise, only $b_{\circ}$ exists.

Proof. See Appendix A.1.

\footnotetext{
${ }^{9}$ The endogeneity of precision relies strictly on the private nature of the price signal. The interested reader can easily go through the previous steps and check that in the case of a public endogenous signal (suppose $\eta_{i}$ is common across islands) its relative precision is independent of the average reaction. More generally, with a homogeneous information set, agents know what others know; therefore, inferring the average expectation would not be a problem.

${ }^{10}$ In game-theoretic terms, $b_{i}(b)$ determines the unique strictly dominant action in response to $b$, a sufficient statistic for the profile of others' actions.
} 


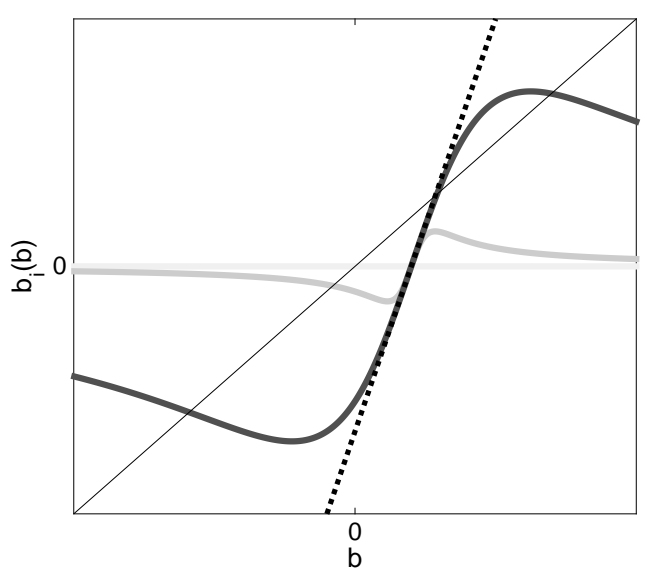

(a) $\mu=1, \alpha=0.7$

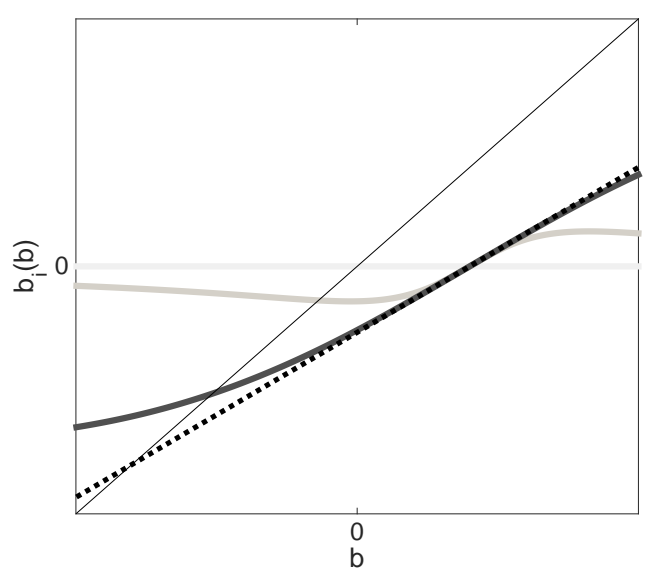

(b) $\mu=0.5, \alpha=0.25$

Figure 2: Fixed-point equation for: $\sigma^{2} \rightarrow \infty$ in solid light gray, $\sigma^{2}=0.5$ in solid gray, $\sigma^{2}=0.02$ in solid dark gray and $\sigma^{2}=0$ in dotted black. Equilibria lie at the intersection with the bisector.

The proof of the proposition can easily be grasped visually from Figure 2. Panels (a) and (b) plot $b_{i}(b)$ in two different cases: $\mu>1-\alpha$ and $\mu<1-\alpha$, respectively, for different values of $\sigma$. In both panels, we observe that when $\sigma^{2} \rightarrow \infty, b=0$ is the unique equilibrium; as $\sigma^{2}$ decreases, the curve approaches the perfect-information (dotted) line obtained at $\sigma=0$; with $\sigma \neq 0$, there is always a sufficiently large absolute value of $b$ such that the optimal weight $b_{i}(b)$ approaches zero. The difference between the two panels is in the slope of the perfect-information line: it cuts the bisector from below if and only if $\mu>1-\alpha$, as in panel (a). Only under this condition does the curve necessarily have two other intersections for a sufficiently small $\sigma .^{11}$

To sum up, the existence of a dispersed-information limit requires two conditions.

First, it must be $\mu>1-\alpha$ for the complementarity induced by the scale factor to be sufficiently strong. When this is the case, prices are more sensitive to demand than to supply conditions. This implies that if producers' reactions to prices increase, the price shrinks more than proportionally, which induces an even stronger individual reaction.

Second, $\sigma$ must be positive but small for the substitutability induced by the precision factor to kick in. On the one hand, $\sigma$ has to be small to let complementarity prevail for a large range so that an equilibrium close to perfect information will be possible. On the other hand, $\sigma$ has to be strictly positive because substitutability does not obtain otherwise.

\footnotetext{
${ }^{11}$ An interesting observation is that the existence of a multiplicity of limit equilibria does not depend on the unboundedness of the domain of $b_{i}$, but only on the slope of the best individual reaction function around the perfect-information limit equilibrium. To see this, suppose that one arbitrarily restricts the support of feasible individual reactions $b_{i}$ in a neighborhood of $b_{\circ}$, namely $\Im\left(b_{\circ}\right) \equiv[\underline{b}, \bar{b}]$. Whenever $\mu>(1-\alpha)$, it is easy to check by a simple inspection of Figure 3 that two other equilibria beyond $b_{\circ}$ would arise as corner solutions. I thank George-Marios Angeletos for directing my attention to this point.
} 
These two conditions together can sustain an equilibrium in which producers react strongly to prices, prices move little and producers are privately uncertain.

The perfect-information limit equilibrium inherits the properties of the equilibrium under perfect information: local prices $r_{i}=(\mu-(1-\alpha)) \theta$ are perfectly correlated and transmit information with infinite precision, that is, $\mathrm{E}\left[\theta \mid r_{i}\right]=\theta$. The picture changes dramatically in a dispersed-information limit.

Proposition 2. The dispersed-information limit equilibria both feature: almost sticky local prices $r_{i} \rightarrow 0$, which transmit information with finite precision

$$
\hat{\tau} \equiv \lim _{\sigma^{2} \rightarrow 0} \lim _{b \rightarrow b_{ \pm}} \tau(b)=\frac{1-\alpha}{\mu-(1-\alpha)} ;
$$

underreactive aggregate expectation

$$
\lim _{\sigma^{2} \rightarrow 0} \lim _{b \rightarrow b_{ \pm}} \int \mathrm{E}\left[\theta \mid r_{i}\right] \mathrm{di}=\frac{1-\alpha}{\mu} \theta
$$

and sizable cross-sectional variance of island-specific expectations

$$
\lim _{\sigma^{2} \rightarrow 0} \lim _{b \rightarrow b_{ \pm}} \int\left(\mathrm{E}\left[\theta \mid r_{i}\right]-\int \mathrm{E}\left[\theta \mid r_{i}\right] \mathrm{di}\right)^{2} \mathrm{di}=\frac{(1-\alpha)(\mu-(1-\alpha))}{\mu^{2}} .
$$

Proof. See Appendix A.2.

Proposition 2 characterizes an equilibrium as the one plotted in panel (c) of Figure 5. The key object is the limit precision (18). This is the exact value at which the impact of productivity on local prices shrinks to such a small component that even tiny local disturbances cause imperfect inference. In this case, even vanishing shocks can generate large cross-sectional heterogeneity in expectations (see (20)). As we will see below, such dispersion in beliefs causes large inefficiencies in the allocation of capital across islands.

\subsection{Welfare and capital misallocations}

As soon as final producers disagree about productivity, the market departs from the unconstrained first best, as the marginal productivity of raw capital is not equalized across islands. Thus, the co-existence of dispersed- and perfect-information limit equilibria entails a case in which a tiny heterogeneity can generate an inefficient allocation without preventing the social optimum from being a possible market outcome.

To make the point formally, let us express welfare as:

$$
Y=\int Y_{(i)} \mathrm{di}=\int e^{\mu \theta} K_{i}^{\alpha} \mathrm{di}=e^{\mu \theta} \bar{K}_{i}^{\alpha} \int e^{\alpha k_{i}} \mathrm{di},
$$

where we make use of (2) and of the log-normal form of the equilibrium. We know that $\bar{K}_{i}=\bar{Z}_{(i)}$ and that

$$
\int Z_{(i)} \mathrm{di}=\bar{Z}_{(i)} e^{\operatorname{var}_{i}\left(z_{(i)}\right) / 2}=Z
$$


which implies $\bar{K}_{i}=Z e^{-\operatorname{var}_{i}\left(z_{(i)}\right) / 2}$, where $\operatorname{var}_{i}\left(z_{(i)}\right)$ denotes the cross-sectional variance of $z_{(i)}{ }^{12}$ Note that, at the limit of $\sigma^{2} \rightarrow 0, \operatorname{var}_{i}\left(k_{i}\right)$ is equal to $\operatorname{var}_{i}\left(z_{(i)}\right)$ according to $(7)$. Moreover, using (9), (13) and (15) we can write

$$
k_{i}=\left(\frac{\mu}{1-\alpha} b-\frac{1}{1-\alpha}\right) r_{i}=\frac{1-\mu b}{1-\alpha}\left(\frac{1-\alpha}{1-\mu b} \theta+\eta_{i}\right)=\theta+\frac{1-\mu b}{1-\alpha} \eta_{i} .
$$

Thus, we have

$$
\begin{aligned}
\lim _{\sigma^{2} \rightarrow 0} Y & =e^{\mu \theta} Z^{\alpha} e^{-\frac{\alpha}{2} \lim _{\sigma^{2} \rightarrow 0} \operatorname{var}_{i}\left(k_{i}\right)} e^{\alpha \theta+\frac{\alpha^{2}}{2} \lim _{\sigma^{2} \rightarrow 0} \operatorname{var}_{i}\left(k_{i}\right)}= \\
& =e^{(\mu+\alpha) \theta} Z^{\alpha} e^{-\frac{\alpha(1-\alpha)}{2} \lim _{\sigma^{2} \rightarrow 0} \operatorname{var}\left(k_{i}\right)},
\end{aligned}
$$

which decreases as the dispersion in the production of local capital $\operatorname{var}_{i}\left(k_{i}\right)$ increases. This is a direct consequence of curvature in the production function measured by $\alpha$.

It is straightforward to note that, in the limit $\sigma^{2} \rightarrow 0$, the first-best allocation $\left(\operatorname{var}_{i}\left(k_{i}\right)=0\right)$ is obtained for any finite $b$. This is not the case for dispersed-information equilibria, in which $\lim _{\sigma \rightarrow 0} b^{2} \sigma^{2}$ is bounded away from zero. Therefore, we have the following.

Proposition 3. The perfect-information limit equilibrium achieves the unconstrained first-best allocation, whereas dispersed-information limit equilibria lead to capital misallocations. In particular, we have

$$
\lim _{\sigma^{2} \rightarrow 0} \lim _{b \rightarrow b_{ \pm}} \operatorname{var}_{i}\left(k_{i}\right)=\frac{\mu-(1-\alpha)}{1-\alpha}>\lim _{\sigma^{2} \rightarrow 0} \lim _{b \rightarrow b_{\circ}} \operatorname{var}_{i}\left(k_{i}\right)=0
$$

Proof. See Appendix A.3.

The proposition establishes that dispersed-information limit equilibria are suboptimal equilibria. Misallocations occur because producers have heterogeneous beliefs about the marginal productivity of capital, although marginal costs are closely uniform.

This result is related to a literature that focuses on imperfect information as a main cause of capital misallocations (see David et al. (2016) for a recent study). The contribution of this simple model is to demonstrate that capital misallocations may stem from imperfect information in economies in which: (i) producers perfectly observe their marginal costs, (ii) forecast errors are driven by fundamentals rather than noise, and (iii) the unconstrained first best is a possible market outcome.

\subsection{Out-of-equilibrium selection}

In this section, I look at the issue of equilibrium selection. I show that, whenever dispersed-information limit equilibria exist, they are stable outcomes of an out-

\footnotetext{
${ }^{12}$ Given that the aggregate supply of raw capital is fixed, the cross-sectional variance $\operatorname{var}_{i}\left(z_{(i)}\right)$ is equal to the unconditional variance $\operatorname{var}\left(z_{(i)}\right)$.
} 
of-equilibrium convergence process in higher-order beliefs, whereas a perfect-information limit equilibrium is not. ${ }^{13}$

The following analysis is inspired by the work on Eductive Learning ${ }^{14}$ by Guesnerie (2005, 1992) and has connections with the usual rationalizability argument used in the Global Games literature (Carlsson and van Damme, 1993; Morris and Shin, 1998). This is a stricter criterion than the stability under adaptive learning (Marcet and Sargent (1989a,b) and Evans and Honkapohja (2001)), which is explored in Appendix A.5.

In the economy, rationality and market clearing are common knowledge among agents, meaning that nobody doubts that individual reactions comply with (17). Nevertheless, this is still not enough to determine which equilibrium, if any, will prevail. For instance, suppose that possible values of $b$ can be restricted to a neighborhood $\Im(\hat{b})$ of an equilibrium characterized by a fixed point $\hat{b}$ of (17). Common knowledge of $\Im(\hat{b})$ implies, as a first conjecture, that the rational individual reactions, and therefore their average, must actually belong to $b_{i}(\Im(\hat{b}))$. But given that $b_{i}(\Im(\hat{b}))$ is also common knowledge, then agents should conclude, in a second-order conjecture, that rational individual reactions, and therefore their average, must actually belong to $b_{i}^{2}(\Im(\hat{b}))$. Iterating the argument, we have that the $\nu$ th.-order conjecture about $b$ belongs to $b_{i}^{\nu}(\Im(\hat{b}))$. Agents can finally conclude that $\hat{b}$ will prevail if the equilibrium is a locally unique rationalizable outcome, as defined below.

Definition 2. A REE characterized by $\hat{b} \in\left(b_{\circ}, b_{+}, b_{-}\right)$is a locally unique rationalizable outcome if and only if

$$
\lim _{\nu \rightarrow \infty} b_{i}^{\nu}(\Im(\hat{b}))=\hat{b},
$$

i.e. $b_{i}$ entails a local contraction around the equilibrium.

From an operational point of view, local uniqueness simply requires that $\left|b_{i}^{\prime}(\hat{b})\right|<1$. The following proposition states the result.

Proposition 4. Whenever dispersed-information limit equilibria exist, they are locally unique rationalizable outcomes, whereas the perfect-information limit equilibrium is not. The perfect-information limit equilibrium is a locally unique rationalizable outcome when it is the only limit equilibrium.

Proof. See Appendix A.4.

To shed some light on the eductive process, let us look back at (17). In a neighborhood of the perfect-information equilibrium value $b_{\circ}$, the precision factor is nearly one,

\footnotetext{
${ }^{13}$ In Appendix A.5 I show that the results are the same also when considering dynamic processes of adaptive learning.

${ }^{14}$ Eductive Learning assesses whether rational expectation equilibria can be selected as locally unique rationalizable outcomes according to the original criterion formulated by Bernheim (1984) and Pearce (1984). The difference here is that I deal with a well-defined probabilistic structure. I consider beliefs about the average weight $b$, rather than beliefs specified directly in terms of price forecasts, as generally assumed by Guesnerie in settings of perfect information.
} 


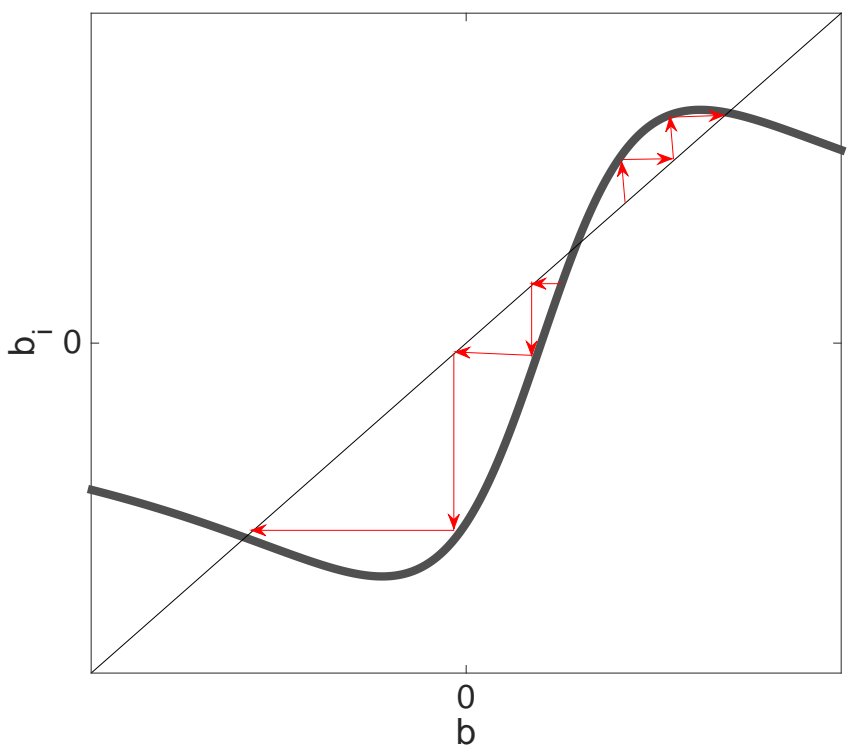

Figure 3: Higher-order belief convergence to dispersed information limit equilibria.

so the dynamics of beliefs are mainly governed by the scale factor. Notice that in the case $\mu>1-\alpha$, the scale factor entails a first-order divergent effect led by high complementarity, i.e., $\lim _{b \rightarrow b_{\circ}} b_{i}^{\prime}(\hat{b})=\mu /(1-\alpha)>1$. In other words, if agents contemplate the possibility that the average reaction is different than the equilibrium value $b_{\circ}$, their best individual reaction must be further from the equilibrium. This evolution corresponds to the divergent dynamics in higher-order beliefs displayed in Figure 3. In this case, the perfect-information limit equilibrium is inherently unstable under higher-order belief dynamics.

Nevertheless, as conjectures about $b$ further diverge from $b_{\circ}$, the reaction of local prices will eventually shrink, to the point where they reach the same order of magnitude as island-specific noise. At that point, a second-order effect driven by the precision factor is activated. Finally, the informativeness of the signal decreases, so that the divergent effect of the scale factor is overturned by an ever lower precision effect, to the point where a new equilibrium emerges. In particular, the sequence of higher-order beliefs eventually enters a contracting dynamic, which, as the proposition proves, converges toward a dispersed-information limit outcome.

\section{The Fragile Neutrality of Money}

This section shows that dispersed-information limit equilibria can have natural microfoundations within the typical setting of monetary DSGE models.

I consider firms that employ local inputs - capital and labor - to produce a variety of a consumption good that imperfectly competes with other varieties. Firms need to set a selling price and install capital before demand realizes. Pricing is not a problem as firms 
observe the prices of their inputs. However, firms are uncertain about the quantity that they will actually produce, and thus, the quantity of capital that is optimal to install. In this respect, they are in the same position as producers in the previous model. The key difference is that here uncertainty does not concern an exogenous state, productivity, but rather an endogenous one, demand, which depends on others' pricing. In particular, as in the famous Lucas (1973), others' pricing is uncertain due to dispersed information about a stochastic change in money supply, which would have no real consequences otherwise. The contribution of the current model is to demonstrate that large non-neutralities may obtain even with a vanishingly small dispersion in fundamentals.

As in the previous model, the core mechanism is embedded in the markets for local capital. The common essential element is that the local price of capital is negatively correlated with the aggregate shock in the case of no information, and positively correlated in the case of perfect information. As we saw, this is the condition that lets, for some finite precision of information, the price signal exhibit a sufficiently small response to the aggregate shock such that vanishing disturbances matter. However, the new setting shows how to naturally obtain this condition without relying on a simultaneous shift in supply and demand (as illustrated in Figure 1), but only in demand. In particular, in response to an increase in money supply, local wages increase and capital demand declines, because of a classical production complementarity. Without any news on the average price level, this complementarity in production pushes down the price of local capital. It is only when producers are sufficiently confident that inflation is a global phenomenon that demand for capital will eventually rise and sustain an increase in local capital prices, as in the neutrality benchmark.

\subsection{A standard monetary model}

\section{Preferences}

Consider an economy composed of a continuum of islands indexed by $i \in(0,1)$. On each island, there are atomistic producers of differentiated consumption goods, who compete for island-specific inputs: local labor and local capital. Local labor is supplied by a representative household, which consumes a bundle of differentiated goods, appreciates the services of real cash holdings, and sells raw capital to intermediate producers of local capital. The household chooses a level of composite consumption $C$, the supply of labor $L_{i}^{s}$ of each type $i \in(0,1)$, and money holdings $M$ in order to solve

$$
\max _{\left\{C_{i}, L_{i}\right\}_{i \in[0,1]}, M}\left\{\frac{C^{1-\psi}-1}{1-\psi}-\int e^{\xi_{i}} L_{i}^{s} \mathrm{di}+\delta \log \frac{M}{P}\right\},
$$

subject to a budget constraint

$$
\frac{R}{P}+\int \frac{W_{i}}{P} L_{i}^{s} \mathrm{di}+\frac{\bar{M}^{s} e^{\theta}}{P} \geq C+\frac{M}{P}
$$


where $\bar{M}^{s} e^{\theta}$ represents a stochastic supply of money; $W_{i}$ is the hourly nominal wage type $i$;

$$
C \equiv\left(\int C_{j}^{\frac{\epsilon-1}{\epsilon}} \mathrm{dj}\right)^{\frac{\epsilon}{\epsilon-1}} \text { and } P \equiv\left(\int P_{j}^{1-\epsilon} \mathrm{dj}\right)^{\frac{1}{1-\epsilon}}
$$

are a composite consumption good and its price respectively; $C_{j}$ is the consumption of the variety $j \in(0,1)$ whose nominal per-unit price is $P_{j} ; \epsilon>1$ is a CES parameter that measures the degree of substitutability of local goods; $\psi$ accounts for the concavity of utility in consumption; $\delta>0$ parameterizes the contribution of real money holdings to utility; and $\xi_{i}$ is an island-specific disutility shock to labor type $i$.

$R$ is the nominal price of one unit of raw capital, which is owned by the household. In a dynamic version of this model, raw capital can be modeled as the legacy of a capital accumulation choice made by the household at the end of the previous period. Nevertheless, what matters to the main results of this paper is that the quantity of raw capital is predetermined at the beginning of each period.

The lack of convexity in labor disutility greatly simplifies exposition; however, I consider the convex case in the main proofs. Let me also remark that the sole role of money in the utility function is to pin down the aggregate price level, which is otherwise undetermined. However, the presence of cash is not essential. As in Woodford (2003), we can define the cashless limit as the economy that obtains by letting $\bar{M}$ and $\delta$ go to zero, while keeping the ratio $\delta / \bar{M}$ fixed to a finite number. In the cashless limit, the average price level remains well determined.

\section{Production}

All producers who operate on the same island solve a symmetric problem. Therefore, from now on, I will label all the variables that concern a representative producer on island $i$ with the same index $i$, and delete the notation for variety $j$. This convention simplifies notation; however, the reader should bear in mind that each producer is a price-taker in her own local input market, while being a competitive monopolist in the global market for consumption.

As usual in models of monopolistic competition, producers set a price and produce to match realized demand. To make our problem meaningful, we will assume here that producers set prices and install capital before demand unfolds. ${ }^{15}$ Once demand realizes, labor is hired to allow market clearing.

Formally, the problem of final producers is

$$
\max _{P_{i}, K_{i}}\left\{P_{i} \mathrm{E}\left[Y_{i} \mid R_{i}, W_{i}\right]-R_{i} K_{i}-W_{i} L_{i}\right\},
$$

under the constraint of a Cobb-Douglas technology with constant returns to scale

$$
Y_{i} \equiv K_{i}^{\alpha} L_{i}^{1-\alpha},
$$

\footnotetext{
${ }^{15}$ If producers were able to observe the demand for their differentiated good when they set prices, then the irrelevance of dispersed information would obtain, as explained by Hellwig and Venkateswaran (2014).
} 
with $\alpha \in(0,1)$, where $K_{i}, L_{i}$, and $Y_{i}$ denote, respectively, the demand for local capital, the demand for local labor, and the produced quantity of a representative producer on island $i$. The presence of $\mathrm{E}\left[Y_{i} \mid R_{i}, W_{i}\right]$ means that producers make their choices before demand realizes while observing the prices of their local inputs.

It is immediately evident that the problem of the final producers above is a generalization of the problem of the final producers in the previous section (see (1) and (2), where, it may be recalled, the price of consumption is normalized to one). Behind the obvious difference - that we are now considering two local inputs - there is another important one. Although in both models producers are uncertain about their actual production, here they produce an island-specific variety that imperfectly competes with other local varieties. As a result, in this setting producers must forecast an endogenous (the demand for their variety), rather than exogenous (productivity), aggregate state that involves guessing others' pricing. We will return to this point later.

Finally, as in the previous section, island-specific capital $K_{i}^{s}$ is supplied by intermediary producers type $i$ who employ a quantity of homogeneous raw capital $Z_{(i)}$ available in a global market at a price $R$. They solve

$$
\max _{Z_{(i)}}\left\{R_{i} K_{i}^{s}-R Z_{(i)}\right\}
$$

under the constraint of a linear technology

$$
K_{i}^{s} \equiv e^{\eta_{i}} Z_{(i)}
$$

where $e^{\eta_{i}}$ is the stochastic island-specific productivity factor. The absence of decreasing returns to scale is adopted for the sake of simplicity; however, I consider the general case in the main proofs. ${ }^{16}$ This specification parallels (3)-(4) in the previous model, except that here we do not assume any particular correlation in productivity shocks. Thus, in this case, the aggregate supply of local capital does not move with the aggregate state.

\section{Shocks}

The economy is hit by i.i.d. aggregate and island-specific disturbances. An aggregate source of randomness,

$$
\theta \sim \mathcal{N}(0,1)
$$

concerns the stock of money available to the representative household. The disutility of working hours type $i$ varies according to

$$
\xi_{i} \sim \mathcal{N}\left(0, \sigma_{\xi}^{2}\right)
$$

\footnotetext{
${ }^{16}$ It is worth noting that, in contrast to the model in Section 2, considering decreasing returns to scale in the intermediate sector of this economy does not alter the conditions for the existence of dispersedinformation limit equilibria.
} 
which introduces differences in labor supply across islands. The productivity of the intermediate sector type $i$ is affected by idiosyncratic productivity shocks

$$
\eta_{i} \sim \mathcal{N}\left(0, \sigma^{2}\right)
$$

where $\eta_{i}$ is an i.i.d. realization across islands. The size of $\sigma^{2}$ represents a measure of the cross-sectional heterogeneity in the rental price of capital across islands.

\section{Timing of actions and information acquisition}

The economy unfolds in three stages.

i) In the first stage, the shocks hit. The household observes the money supply shock and the shocks to labor disutility. Each intermediate producer observes her own productivity shock.

ii) In the second stage, the markets for raw capital and local capital open and clear simultaneously. On each island, final producers observe the equilibrium price of their local inputs, install capital, and set their selling price.

iii) In the last stage, demand realizes and final producers hire the quantity of labor needed to clear the market.

The absence of convexity in the disutility of labor allows a local equilibrium wage, which is observable in the second stage, to emerge irrespective of the quantity of working hours, which are traded in the third stage. To allow quantity adjustments otherwise, one can assume that producers observe the labor supply schedule posted by the household. ${ }^{17}$ This is informationally equivalent to observing the equilibrium wage. The most general case is fully worked out in the main proofs in Appendix B.1.

\subsection{Equilibria}

\section{Definition of equilibrium and first-order conditions}

As in the previous section, I restrict the analysis to equilibria with a log-normal representation that, in this model as well, obtains with no approximation. A formal definition of an equilibrium follows below.

Definition 3. A log-normal rational expectation equilibrium is a distribution of prices $\left\{\left\{R_{i}, W_{i}, P_{i}\right\}_{i \in[0,1]}, R\right\}$, quantities $\left\{C_{i}, Y_{i}, M, L_{i}, L_{i}^{s}, K_{i}, K_{i}^{s}, Z_{(i)}\right\}_{i \in[0,1]}$ and expectations $\left\{\mathrm{E}\left[Y_{i} \mid R_{i}, W_{i}\right]\right\}_{i \in[0,1]}$, contingent on the stochastic realizations $\left(\theta,\left\{\xi_{i}, \eta_{i}\right\}_{i \in[0,1]}\right)$, such that:

- (optimality) agents optimize their actions according to the prices they observe;

\footnotetext{
${ }^{17} \mathrm{~A}$ more cumbersome solution is to introduce a third production factor whose market opens and clears in the last stage, and let labor be traded in the second stage.
} 
- (market clearing) the market for the raw capital clears, $\int Z_{(i)} d i=1$; demand and supply in local markets match, $L_{i}=L_{i}^{s}$ and $K_{i}=K_{i}^{s}$; final markets clear, $Y_{i}=C_{i}$; and money demand equals supply, $M=\bar{M}^{s} e^{\theta}$;

- (log-normality) prices and quantities are log-normally distributed.

The first condition ensures that agents optimize their actions using rationally the information conveyed by the equilibrium prices they observe. The requirement of a lognormal equilibrium allows the tractability of aggregate and island-specific relations.

The household chooses a level of aggregate and island-specific consumption, islandspecific working hours and future money holdings such that:

$$
\begin{aligned}
\Lambda & =C^{-\psi}, \\
C_{i} & =\left(\frac{P_{i}}{P}\right)^{-\epsilon} C, \\
e^{\xi_{i}} & =W_{i} \frac{\Lambda}{P}, \\
\frac{\Lambda}{P} & =\frac{\delta}{\bar{M}^{s} e^{\theta}},
\end{aligned}
$$

respectively, where $\Lambda$ is the Lagrangian multiplier associated with the budget constraint of the household and we have already used $M=\bar{M}^{s} e^{\theta}$. Intermediate producers supply any quantity of local capital provided

$$
R_{i}=e^{-\eta_{i}} R
$$

that is, the price of the local capital equals the cost of the raw capital augmented by the local productivity shock. (40) is the only equilibrium relation yielded by the presence of intermediate production. Its role is to generate heterogeneity in the cost of capital. ${ }^{18}$ Without learning from prices, such heterogeneity would be completely irrelevant to the aggregate behavior of the economy; on the contrary, with learning from prices, it may have major consequences no matter how small it is.

The household and the intermediate producers can solve their problems without any uncertainty, as they directly observe all the relevant variables. This is not the case for final producers. A producer type $i$ sets a price for her own variety, charging a markup $\epsilon /(\epsilon-1)$ on her own nominal marginal cost; that is

$$
P_{i}=\frac{\epsilon}{\epsilon-1} \frac{R_{i}^{\alpha} W_{i}^{1-\alpha}}{(1-\alpha)^{1-\alpha} \alpha^{\alpha}}
$$

which is a standard outcome of monopolistic competitive economies (see, for example, Christiano et al. (2005) page 10).

\footnotetext{
${ }^{18}$ This feature shares the spirit of a strand of New Keynesian literature that stresses the importance of capital market segmentation for the amplification of money non-neutralities. See, for example, Woodford (2005) and Altig et al. (2011) for models with firm-specific capital and Carvalho and Nechio (2016) for an extension to sectoral-specific capital.
} 
Then, for a given level of expected production $\mathrm{E}\left[Y_{i} \mid R_{i}, W_{i}\right]$ and given input prices $R_{i}$ and $W_{i}$, a producer type $i$ chooses capital $K_{i}$ to minimize total expenditure $K_{i} R_{i}+W_{i} L_{i}$ subject to a technology constraint $\mathrm{E}\left[Y_{i} \mid R_{i}, W_{i}\right]=K_{i}^{\alpha} L_{i}^{1-\alpha}$. Simple algebra yields

$$
K_{i}=\left(\frac{\alpha W_{i}}{(1-\alpha) R_{i}}\right)^{1-\alpha} \mathrm{E}\left[Y_{i} \mid R_{i}, W_{i}\right]
$$

which determines the optimal demand for capital for a given level of expected production. Finally, the demand for local labor is determined in the third stage, such that $C_{i}=Y_{i}=$ $K_{i}^{\alpha} L_{i}^{1-\alpha}$, i.e., final producers hire labor to let production match demand. Hence, the optimal mix of inputs obtains only in the case of perfect information $\mathrm{E}\left[Y_{i} \mid R_{i}, W_{i}\right]=Y_{i}$, whereas incomplete information causes ex-post losses.

The conditions for money neutrality can be easily checked by inspection of (36)-(42). In particular, an equilibrium in which money is neutral is an equilibrium in which the ratio between any price and $e^{\theta}$ remains constant for whatever realization of $\theta$, so that first order conditions are not affected by $\theta$. In such a case, the real allocation is determined irrespective of any fluctuation in money supply. However, this requires that expected demand does not vary with prices, which is not necessarily true in our model of learning from prices.

Importantly, notice that expectations appear in (42) but not in (41). In fact, observing $R_{i}$ and $W_{i}$ is sufficient for final producers to set an optimal price, i.e. pricing is completely frictionless. On the other hand, to set the optimal mix of inputs, producers need to figure out how much they will produce. Therefore, exactly as in the model in Section 2, learning from prices only impacts producers' demand for capital. Let us examine the functioning of capital markets more closely.

\section{Inspecting capital markets}

According to (37), the actual demand for local good $Y_{i}$ depends on (i) the price of the variety $P_{i}$ chosen by producers, (ii) the average price level and (iii) total demand. Thus, one can write the expectation of producers on island $i$ as

$$
\mathrm{E}\left[Y_{i} \mid R_{i}, W_{i}\right]=\mathrm{E}\left[X^{\epsilon} \mid R_{i}, W_{i}\right] P_{i}^{-\epsilon},
$$

where

$$
X \equiv Y^{\frac{1}{\epsilon}} P
$$

is the endogenous common component of each island-specific demand, which producers do not observe at the time of their choices. In contrast to the model in Section 2, here producers are uncertain about an aggregate endogenous variable which is determined by the simultaneous pricing choice of all producers. This uncertainty arises because producers cannot observe $\theta$ directly: knowing $\theta$ is equal to knowing $X$. 
(a)

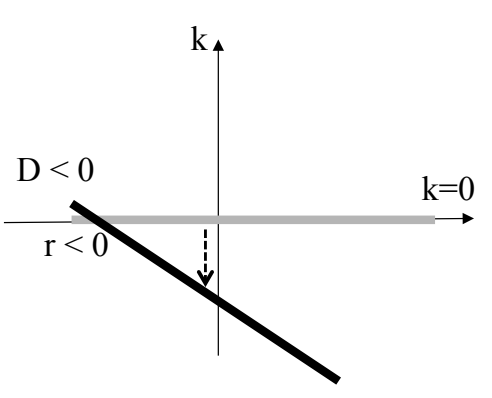

(b)

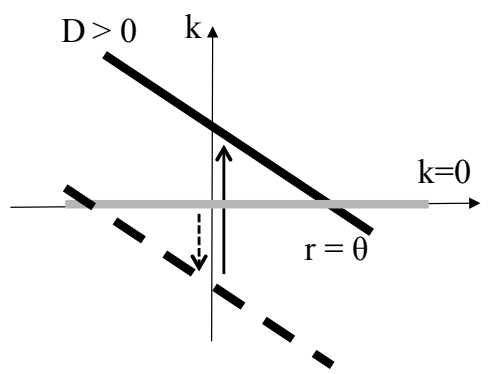

(c)

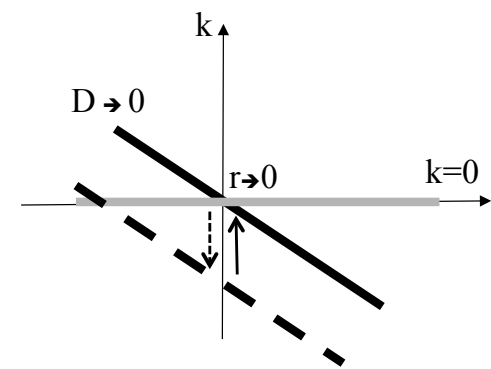

Figure 4: The effect of a positive $\theta$ on the average supply (light gray) and average demand (dark gray) in capital markets. Arrows illustrate the shifts in demand in three cases: no information (panel a), perfect information (panel b) and partial information (panel c). Solid (resp. dashed) arrows denote shifts that (resp. do not) depend on expectations. In panel (b) and (c) the thick dashed line represents the no-information demand.

Combining (42),(41) and (43), one can express local capital demand in log-deviation terms $^{19}$ as

$$
k_{i}=\epsilon \mathrm{E}\left[x \mid r_{i}, w_{i}\right]-(\epsilon-1)(1-\alpha) w_{i}-(1+(\epsilon-1) \alpha) r_{i},
$$

where

$$
w_{i}=\theta+\xi_{i}
$$

is obtained after plugging (39) into (38). Demand for capital depends negatively on the local wage. This is due to a complementarity in production: an increase in local wages depresses the demand for local capital.

The average demand for local capital is given by

$$
k \equiv \int k_{i} \mathrm{di}=\underbrace{\epsilon \mathrm{E}\left[x \mid r_{i}, w_{i}\right] \mathrm{di}-(\epsilon-1)(1-\alpha) \theta}_{\equiv D}-(1+(\epsilon-1) \alpha) r
$$

which is the analog of (10) with a major difference: the demand shifter $D$ now depends on realization of the aggregate state $\theta$. In particular, $\theta$ shows up as a change in money supply that directly affects all wages.

In Figure 4 we illustrate how, for a given positive realization of $\theta$, the equilibrium in the capital market depends on final producers' expectations. We use the same conventions as in Figure 1 so that it is easy to compare the two.

The downward-sloping line denotes average demand (47), the horizontal line average supply. In contrast to the case depicted in Figure 1, here the average supply of capital

\footnotetext{
${ }^{19}$ As before, log-normality of the equilibrium implies that $X$, as with any other variable in the model, follows a log-normal rule $X=\bar{X} e^{x}$, where $x \sim \mathcal{N}(0, \operatorname{var}(x))$ is the stochastic log-deviation of $X$ from its deterministic component $\bar{X}$. The stochastic steady state of $X$ is given by $\bar{X}^{\frac{1}{2} \operatorname{var}(x)}$.
} 
does not move, i.e., $k=0$. On the other hand, a positive $\theta \operatorname{shifts} D$ down as soon as local wages increase (dotted arrow); importantly, the effect of wages on capital demand is independent of producers' expectations. This case is illustrated in panel (a), where we shut down any effect of information fixing $\mathrm{E}\left[x \mid r_{i}, w_{i}\right]=0$. In this configuration $\theta$ and $r$ are negatively correlated, as in the corresponding panel in Figure 1.

The perfect-information scenario is illustrated in panel (b), where we fix $\mathrm{E}\left[x \mid r_{i}, w_{i}\right]=\theta$ instead. ${ }^{20}$ Higher local prices entail more expensive local inputs, but a higher expected $x$ overturns this effect (solid arrow) up to the point at which the average equilibrium price increases by $\theta$, as in the neutrality benchmark. In this case, as in the analogous case in Figure 1, $\theta$ and $r$ are positively correlated.

Therefore, the correlation of $r$ with $\theta$ is: negative in the case of no information, and positive in the case of full information. As before, by continuity, ${ }^{21}$ this implies that there is a finite value of precision for which the upward shift due to information (solid arrow) is just sufficient to offset the effect of a change in local wages (dotted arrow). This situation is plotted in panel (c). At that point, the average response of local prices to the aggregate shock can be so small that even a tiny idiosyncratic noise in local prices renders them poorly informative about $\theta$. This is exactly the same basic mechanism that underlies the results in the previous section.

\section{Learning from prices}

Here, I recover the law of motion for the price signals $w_{i}$ and $r_{i}$ and the uncertain variable $x$. With these elements, we can work out the signal extraction problem of producers.

The wage is simply given by (46). Thus, observing the wage provides a private signal about $\theta$ with exogenous precision $\sigma_{\xi}^{-2}$.

The price of capital $r_{i}$ is obtained in two steps. First, we plug the market clearing condition $k=k^{s}=0$ into (47) to get an expression for $r$. Then we use $r_{i}=r-\eta_{i}$ (from (40)) to get

$$
r_{i}=\phi \int \mathrm{E}\left[x \mid r_{i}, w_{i}\right] \mathrm{di}+(1-\phi) \theta-\eta_{i}
$$

where

$$
\phi \equiv \frac{\epsilon}{1+(\epsilon-1) \alpha}>1
$$

As in our simple model (see (12)), $r_{i}$ constitutes a private endogenous signal that reacts positively to the average expectation and negatively to the aggregate fundamental. However, in this economy, $r_{i}$ always reacts more to the average expectation than to the

\footnotetext{
${ }^{20}$ Here we anticipate that under perfect information, $x$ is equal to $\theta$; in particular, this is the case for money neutrality with $y=0$ and $p=\theta$. We will formally prove the existence of this equilibrium in the main proposition.

${ }^{21}$ For the continuity argument to hold we need, given a positive $\theta, x$ to be monotonically increasing in the precision of information about $x$ that final producers have. We will formally prove this property in the next subsection.
} 
aggregate fundamental, as $\phi>1$ occurs for all feasible parameter values. Moreover, here final producers form an expectation about an endogenous aggregate state $x$ - which is a function of $\theta$ - and not about $\theta$ directly.

The actual law of motion of $x$ is obtained plugging (36) into (39) to get $\psi y=\theta-p$, and then using $x=y / \epsilon+p$, according to (44), to derive $\psi \epsilon(x-p)=\theta-p$. To express $p$ as a function of $\theta$ and $r$, use (41), i.e. $p_{i}=\alpha r_{i}+(1-\alpha) w_{i}$, to recover $p=\alpha r+(1-\alpha) \theta$. Finally, given (48), we get

$$
x=\beta \int \mathrm{E}\left[x \mid r_{i}, w_{i}\right] \mathrm{di}+(1-\beta) \theta
$$

where

$$
\beta \equiv \frac{\alpha\left(\epsilon-\psi^{-1}\right)}{1+(\epsilon-1) \alpha}<1
$$

Note that (50) has a structure similar to the average of (48), except that the strength of the expectational feedback is smaller than one. This feature prevents a switch in the sign of the correlation between $x$ and $\theta$, which is instead the key feature of the price signal. ${ }^{22}$

The signal extraction problem spelled out above generalizes the problem studied in the previous section along two dimensions. First, producers also observe a local wage that provides additional private information. Second, the uncertain variable is now endogenous, that is, it also reacts to expectations. The current setting nests the previous one in the case $\sigma_{\xi}=\infty$ and $\beta=0$.

Finally, it is worth commenting on the role of the various structural parameters. A higher $\epsilon$ or lower $\alpha$ magnify $\phi$, making $1-\phi$ more negative; that is, given an increase in wages - i.e. a positive $\theta$ - the price of local capital decreases more when production is more labor-intensive (the input externality is stronger) or when consumption is more elastic to final prices (competition is tighter). The degree of convexity of utility $\psi$ decides the sign of $\beta$. In particular, when $\psi$ is small, adjustments in the final market occur mainly in quantities rather than in prices. In this case, in response to an excess of optimism on the part of producers, the actual $x$ becomes negative, as a large drop in consumption is caused by a small increase in prices; it is positive otherwise when a large increase in prices causes a small drop in consumption.

\section{Characterization and existence of an equilibrium}

The requirement of a log-normal equilibrium implies that any variable in the model, including expectations, is distributed log-normally. We can characterize an equilibrium in terms of a profile of final producers' expectations about $x$ as follows.

\footnotetext{
${ }^{22}$ Most of the literature about endogenous information structures investigates the effect of private signals about the average action (as for example in Amador and Weill (2012)), which, in this context, is equal to private signals about $x$. By construction, these signals would not exhibit a sufficient reaction to the average expectation to generate the multiplicity that I document in this paper.
} 
Proposition 5. Given a profile of weights $\left\{\mathrm{e}_{\theta}, \mathrm{e}_{\xi}, \mathrm{e}_{\eta}\right\}$ such that log-normal expectations for final producers are described by

$$
\mathrm{E}\left[X \mid R_{i}, W_{i}\right]=\overline{\mathrm{E}} e^{\mathrm{E}\left[x \mid r_{i}, w_{i}\right]},
$$

where $\overline{\mathrm{E}}=\bar{X}^{\frac{1}{2} \operatorname{var}\left(x \mid r_{i}, w_{i}\right)}$ with

$$
\mathrm{E}\left[x \mid r_{i}, w_{i}\right]=\mathrm{e}_{\theta} \theta+\mathrm{e}_{\xi} \xi_{i}+\mathrm{e}_{\eta} \eta_{i}
$$

there is then a unique log-normal conditional deviation and a unique steady state for each variable in the model.

Proof. See Appendix B.1.

In practice, an equilibrium is characterized by a distribution of producers' expectations about $x$, the stochastic aggregate component of demand. Each individual expectation type $i$ is conditional on observation of $w_{i}$ and $r_{i}$, denoting the stochastic log-components of $W_{i}$ and $R_{i}$ respectively. Both are log-normal functions of the shocks, so producers' expectations must be a linear function of the two signals. In particular, all agents use the rule

$$
\mathrm{E}\left[x \mid r_{i}, w_{i}\right]=b_{i}\left(\phi \int \mathrm{E}\left[x \mid r_{i}, w_{i}\right] \mathrm{di}+(1-\phi) \theta-\eta_{i}\right)+a_{i}\left(\theta+\xi_{i}\right),
$$

where $a_{i}$ and $b_{i}$ denote the weights put by agent $i$ on the local wage and the price of local capital respectively. Let us denote the average weights by $a$ and $b$. As in the previous section, a log-normal equilibrium has to be symmetric. Hence, a profile of the optimal weights given to these two pieces of information maps into a profile of weights $\left\{\mathrm{e}_{\theta}, \mathrm{e}_{\xi}, \mathrm{e}_{\eta}\right\}$. The characterization of an equilibrium follows straightaway, once the requirement of rational expectations is imposed.

Definition 3 A log-normal rational expectation equilibrium is characterized by a profile of weights $\left\{\mathrm{e}_{\theta}, \mathrm{e}_{\xi}, \mathrm{e}_{\eta}\right\}$ such that (54) are rational expectations of (50) conditional on (46) and (48), with

$$
\mathrm{e}_{\theta}=\frac{(1-\phi) b+a}{1-b \phi}, \mathrm{e}_{\xi}=a, \mathrm{e}_{\eta}=b
$$

being the corresponding weights in (53).

In other words, the number of equilibria in the model corresponds to the number of solutions of the signal extraction problem. In the cases of no information $\left(\sigma^{2} \rightarrow \infty\right.$ and $\left.\sigma_{\xi}^{2} \rightarrow \infty\right)$ and full information $\left(\sigma^{2}=0\right)$, the economy has a unique equilibrium characterized by $(a, b)=(0,0)$ and $(a, b)=(0,1)$ respectively. The following proposition establishes the existence of multiple equilibria at the limit of a vanishing dispersion of local prices for capital, i.e. when $\sigma^{2} \rightarrow 0$.

Proposition 6. Consider the problem of agents forecasting (50) conditionally on the information set $\left\{r_{i}, w_{i}\right\}$ given by (46) and (48). If the variance of preference shocks $\sigma_{\xi}^{2}$ satisfies

$$
\sigma_{\xi}^{-2}<\hat{\tau}=\frac{\phi-1}{1-\beta}
$$


then in the limit of no productivity shocks, $\sigma^{2} \rightarrow 0$, there are:

- a unique perfect-information limit equilibrium, characterized by $a=0$ and $b=1$, in which the neutrality of money holds, i.e.

$$
r=p=\theta \text { and } y=0,
$$

for any realization of $\theta$;

- and two dispersed-information limit equilibria, characterized by $a=(1-\beta)\left(\phi \sigma_{\xi}^{2}\right)^{-1}$ and $b=b_{ \pm}$with $\lim _{\sigma^{2} \rightarrow 0} b_{ \pm}^{2} \sigma^{2}=\hat{\tau}^{-1}\left(\sigma_{\xi}-\hat{\tau}^{-2}\right) \sigma_{\xi}^{-2}$, in which shocks to the value of money produce real effects; in particular,

$$
r \rightarrow 0, p=(1-\alpha) \theta \text { and } y=\alpha \psi^{-1} \theta
$$

for any realization of $\theta$.

Otherwise, only the perfect-information limit equilibrium exists.

Proof. See Appendix B.2.

The perfect-information limit equilibrium implies that money is neutral. To see this formally, note that under perfect information (50) implies $x=\theta$, which, according to (48), gives $r=\theta$. Because of (41), i.e. $p_{i}=\alpha r_{i}+(1-\alpha) w_{i}$, we also know that under perfect information $p=\theta$. Finally, given the definition of $x$, we have $y=0$; that is, when prices fully react to changes in aggregate money, then real variables, including expected demand, are unaffected.

On the contrary, in a dispersed-information limit equilibrium, a positive $\theta$, i.e. an increase in the stock of money, has real expansionary effects in line with the original Phelps-Lucas' island model. The novelty here is that even a tiny dispersion in fundamentals - in this case, productivity shocks - can generate large departures from the neutrality benchmark. A general discussion of the impact of money shocks is postponed to Section 3.3. Here, it is worth discussing some new insights about the existence of a dispersed-information limit.

The proof of Proposition 6 mimics that of Proposition 1; the fixed point equation that characterizes the equilibrium $b$ is a cubic with the exact same qualitative features as before. The difference is that now the shape of the best equilibrium weight is also influenced by $\sigma_{\xi}$ and $\beta$. In particular, the presence of a finite $\sigma_{\xi}$ generates a new condition (55), which relates to $\hat{\tau}$. Let me discuss the economic interpretation of this new constraint.

$\hat{\tau}$ is the key equilibrium object for the emergence of a dispersed-information limit. Analogous to (16), $\hat{\tau}$ defines the value of information precision for which the sensitivity of $r_{i}$ to $\theta$ shrinks to a sufficiently small number. In a dispersed-information limit, such a threshold value is obtained as the sum of the precision conveyed jointly by $r_{i}$ and $w_{i}$. In particular, for a given precision of wages, the precision of $r_{i}$ will endogenously adjust to arrive at the sum equal to $\hat{\tau}$. The new condition (55) is simply the result of an obvious 
non-negativity constraint: if the precision of wages, which is exogenous, is already larger than $\hat{\tau}$, there cannot be a negative adjustment.

In terms of Figure 4, a violation of (55) implies that the information conveyed by wages is sufficiently precise to completely overturn the initial downward shift due to production complementarity. In such a case, the net effect of a rise in the local wage is an increase, rather than a decrease, in demand. Given that the information conveyed by the price of capital further increases demand, the equilibrium $r$ can never be pushed back close to zero, which prevents the existence of a dispersed-information limit equilibrium.

Condition (55) should be interpreted more generally as a constraint on the dispersion of information other than $r_{i}$. In Appendix B.4 I prove that (55) is still the relevant condition when considering extended versions of the additional private signal (46), which can include correlated noise and/or have endogenous precision (i.e. the signal also reacts to the average expectation). In fact, the existence of a dispersed-information limit depends only on the possibility that the variance of the common component of the endogenous signal shrinks to a sufficiently small number, regardless of the presence of common noise in it.

Also in this model, the existence of a dispersed-information limit implies that a vanishing dispersion in prices can cause large private uncertainty. The following proposition provides a measure of the cross-sectional dispersion of beliefs in the economy.

Proposition 7. In contrast to the perfect-information equilibrium, the dispersed-information limit equilibria feature non-zero cross-sectional variance of expectations:

$$
\lim _{\sigma^{2} \rightarrow 0} \lim _{b \rightarrow b_{ \pm}} \int\left(\mathrm{E}\left[x \mid r_{i}, w_{i}\right]-\int \mathrm{E}\left[x \mid r_{i}, w_{i}\right] \mathrm{di}\right)^{2} \mathrm{di}=\frac{(1-\beta)(\phi-1)}{\phi^{2}}
$$

Proof. See Appendix B.3.

One important result of this richer model is that, due to producers' confusion, idiosyncratic preference shocks $\xi_{i}$ also play a role in pinning down producers' expectations. This does not occur at the perfect-information limit. Specifically, the contribution of idiosyncratic preference shocks to the overall cross-sectional volatility of expectations is given by $a^{2} \sigma_{\xi}^{2}$, which converges toward (56) as (55) becomes binding. We will discuss how such dispersion in beliefs is transmitted to the economy in Section 3.3.

It is worth noting that the equilibrium value of $a$ is inversely proportional to $\sigma_{\xi}^{2}$. This effect occurs because the higher the $\sigma_{\xi}^{2}$, the lower the information transmitted by the local wage. This is an essential feature of learning from prices. Note that rational inattention (Mackowiak and Wiederholt, 2009) implies the opposite comparative statics: the higher the variance of idiosyncratic shocks, the higher the attention agents are willing to pay to them, and so the higher the sensitivity of expectations to idiosyncratic conditions.

Lastly, I show in Appendix B.5 that, in this case as well, whenever dispersed-information limit equilibria exist, they obtain as the only stable equilibrium outcome of a convergence process in higher-order beliefs and adaptive learning. 


\subsection{Out-of-the-limit: a numerical exploration}

The aim of this section is threefold: (i) to illustrate the properties of limit equilibria documented above, (ii) to show that the properties of equilibria at the limit are inherited by continuity by equilibria outside the limit, and (iii) to argue that the economic implications of the model are consistent with widely accepted findings on the real effects of monetary shocks.

I will focus on a version of the model (fully worked out in Appendix B.1) that includes convex disutility of labor. In this case, the objective of the household reads

$$
\frac{C^{1-\psi}-1}{1-\psi}-\int e^{\xi_{i}} \frac{\left(L_{i}^{s}\right)^{1+\gamma}}{1+\gamma} \operatorname{di}+\delta \log \frac{M}{P},
$$

instead of (27), where $\gamma>0$ is the inverse of the Frisch elasticity of labor. As we will see, discussing $\gamma$ is useful to get a better understanding of how producers' heterogeneous beliefs may affect the final allocation.

\section{The impact of a money shock on the aggregate economy}

Let us look first at the impact of aggregate shocks on aggregate variables, as illustrated in Figure 5. On the y-axis, we measure the equilibrium reaction of four variables $p, y, r$ and $w$ - conditional on a unitary realization of $\theta$. On the $\mathrm{x}$-axis, we measure the dispersion of productivity shocks $\sigma$. Our numerical example is obtained setting $\epsilon=8$, $\psi=2, \alpha=0.33$ and $\sigma_{\xi}^{2}=0.4$ for two values of $\gamma: 0$ denoted by a solid line and 0.75 denoted by a dashed line. For the moment, let us focus on the solid line and postpone discussion of the effect of a positive $\gamma$.

For each value of $\sigma$, either one or three equilibrium values exist. In particular, a multiplicity exists for $\sigma$ sufficiently small but not zero; otherwise, a unique equilibrium exists. The dispersed-information limit is represented by the point of tangency of the curves with the y-axis. In general, we can distinguish three loci: a set of equilibria that for $\sigma \rightarrow 0$ converges to the perfect-information equilibrium (denoted in light gray), and two sets of equilibria that for $\sigma \rightarrow 0$ converge to a dispersed information limit (denoted in dark gray). Notice that only one set of equilibria exists for a large enough $\sigma$.

At the perfect-information limit equilibrium, all prices react one-to-one to the money shock, whereas average real quantities remain unchanged; that is, $p=w=r=\theta$ and $l=y=0$. In contrast, at the dispersed-information equilibria, shocks to money have real effects: aggregate price and output go up together, although the price level rises less than with money neutrality. In particular, the lack of reaction of the local price for capital to the aggregate shock feeds through into lower-than-optimal final prices, which boost actual demand.

Let us now contrast the solid and dashed lines to discuss the role of the convexity of labor disutility. A higher $\gamma$ increases the sensitivity of local wages to local labor. Therefore, when producers overestimate adverse local conditions, they expect to hire less labor at lower wages. In turn, lower expected wages push the local selling price further 

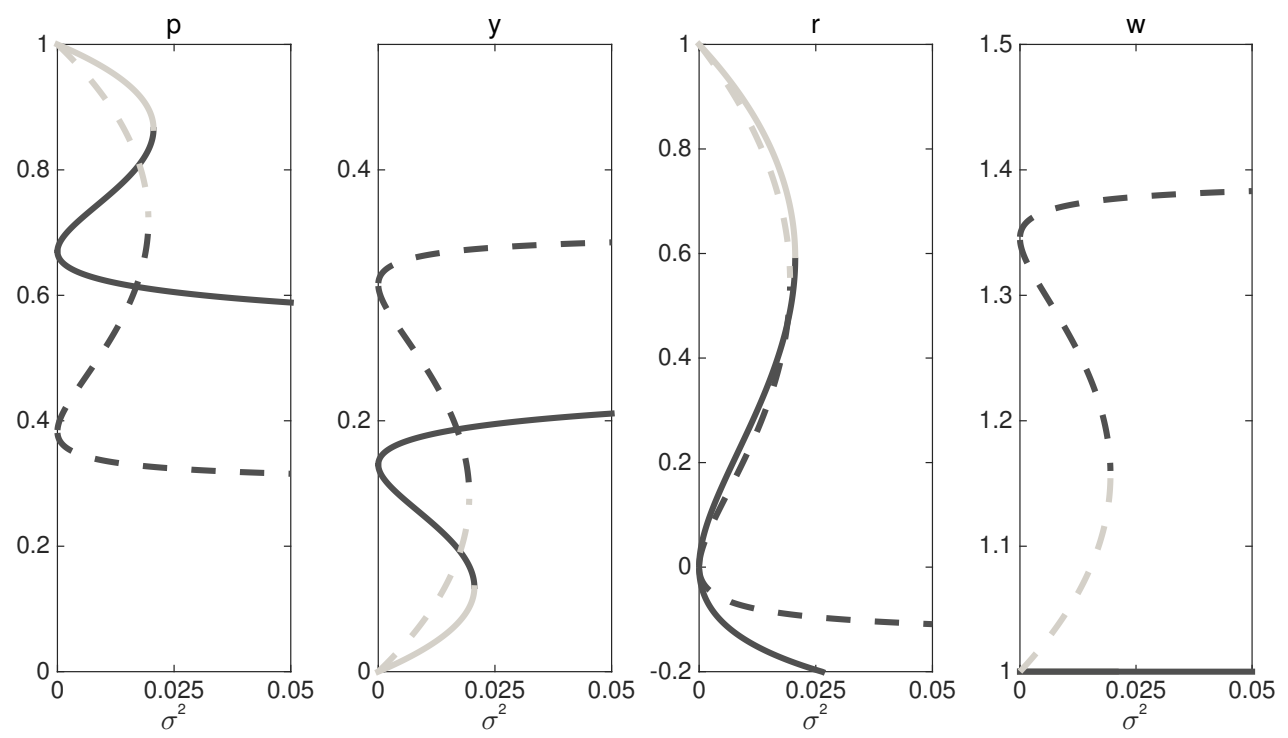

Figure 5: The reaction of aggregate variables to $\theta=1$ for $\epsilon=8, \alpha=0.33, \psi=2$ and $\sigma_{\xi}^{2}=0.4$ for: $\gamma=0$ (solid) and $\gamma=0.75$ (dashed).

down, which ex post induces higher demand. Once demand realizes, producers are forced to hire more labor than expected, which pushes up the actual wage, as plotted in the last panel. In sum, a higher $\gamma$ magnifies the real effects of a money shock on the aggregate economy, although these also obtain in the case $\gamma=0$.

The real effects of money shocks in this economy are consistent with the original insights of the Lucas island model (Lucas, 1972) and the medium-term impact of monetary shocks discussed in seminal works such as Woodford (2003), Christiano et al. (2005) and Smets and Wouters (2007), among others. In contrast to traditional models of price rigidities, in the current setting the non-neutrality of money obtains although producers perceive their marginal costs precisely and are not constrained in their price setting.

\section{The impact of idiosyncratic shocks on local economies}

In Figure 6, a solid line denotes the cross-sectional volatility of $p_{i}, y_{i}, r_{i}$ and $w_{i}$ as a function of $\sigma^{2}$. The dotted line represents the fraction of overall cross-sectional volatility due only to idiosyncratic preference shocks.

I use the same convention as in Figure 5 to denote the three loci of equilibria. The numerical example is obtained setting $\epsilon=8, \psi=2, \alpha=0.33$ and $\sigma_{\xi}^{2}=0.4$ and $\gamma=0.75$.

It is important now to focus on a case with $\gamma>0$ because belief dispersion can affect the cross-section of final allocations as long as it generates differences in local wages. In fact, with $\gamma=0$, the dispersion of prices is given exogenously by the dispersion of local wages, $\sigma_{\xi}^{2}$, and the local price for capital, $\sigma^{2}$. In contrast, with $\gamma>0$, the dispersion of beliefs about expected demand affects the dispersion of expected wages, and so the 

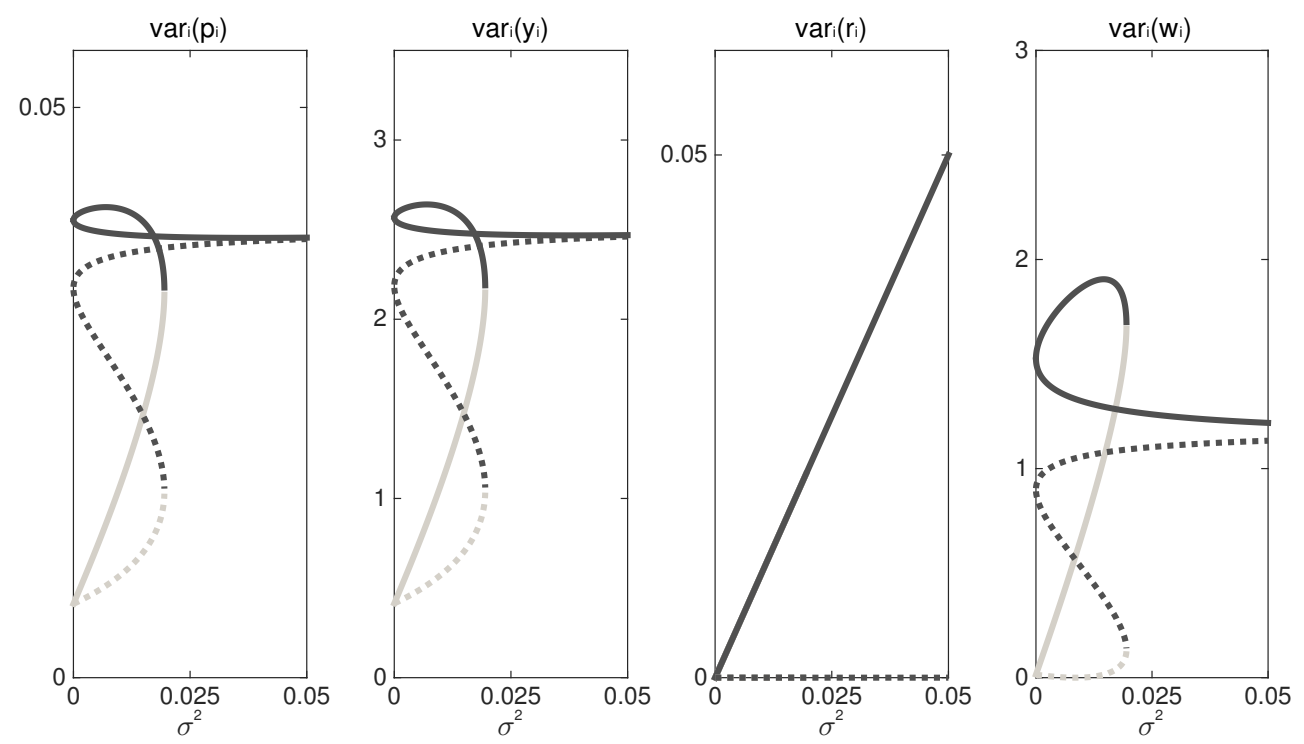

Figure 6: Cross-sectional dispersion of $p_{i}, y_{i}, r_{i}$ and $w_{i}$ for $\epsilon=8, \alpha=0.33, \psi=2, \sigma_{\xi}^{2}=0.4$ and $\gamma=0.75$. The dotted line denotes the contribution of idiosyncratic preference shocks to overall dispersion.

dispersion of final prices, generating dispersion in actual demand across islands.

At the perfect-information limit equilibrium, idiosyncratic preference shocks explain all the cross-sectional dispersion in the economy. At the dispersed-information limit equilibria, idiosyncratic preference shocks do not explain all the cross-sectional dispersion, although their absolute contribution is higher. In other words, infinitesimally small productivity shocks are responsible for a sizable fraction of price-quantity dispersion, and, at the same time, the impact of shocks to disutility is amplified compared with the perfectinformation scenario.

This finding is in line with the evidence put forward by Boivin et al. (2009) that, although prices respond little to aggregate shocks, they respond strongly to idiosyncratic ones, so that the latter explain most overall price volatility in final markets.

The figures also demonstrate that a tiny cross-sectional variance of productivity shocks can generate sizable dispersion of prices and quantities in the economy. In this case, an external observer looking for the source of firms' disagreement could easily miss its fundamental nature. This insight offers a different interpretation for the price dispersion puzzles documented, for example, by Eden (2013) and Kaplan and Menzio (2015), for which search and matching models (see Burdett and Judd (1983)) generally offer an alternative explanation. In particular, non-measurable dispersion in fundamentals can generate sizable price dispersion in final markets without assuming any search friction. 


\section{Conclusion}

This paper sets out the conditions under which the introduction of an arbitrarily small degree of dispersion in fundamentals can feature price rigidity as an equilibrium outcome. When agents learn from local prices, no matter how small their dispersion, the tension between the allocative and informative roles of prices can dramatically alter their functioning. I have shown that this mechanism is naturally reproduced by a typical input demand function, and so potentially pertains to a large class of macro models. In particular, I have presented a standard monetary model with flexible prices in which, with no heterogeneity, a unique equilibrium exists in which money neutrality holds. I have shown that the introduction of vanishingly small heterogeneity in input markets can generate another equilibrium in which prices underreact to aggregate monetary shocks and overreact to idiosyncratic shocks. This equilibrium, which I tagged as a dispersedinformation limit equilibrium, has strong stability properties, in contrast to the perfectinformation limit equilibrium.

I leave some important issues for future research. The present model is essentially static in nature, as current realizations are not informative about the future course of the economy. When this is not the case, agents gather correlated information over time. The extent to which non-neutralities can persist in a economy in which agents learn from current and past prices is a question that hopefully this paper will help to address in the near future. Finally, despite the model's ability to naturally generate nominal rigidities and even price stickiness in the limit, it cannot reproduce the frequency of

price adjustments, which is the focus of most of the microeconometric literature on price setting. 


\section{Appendix}

\section{A A simple real economy}

\section{A.1 Proof. of proposition 1}

With $\sigma^{2}=0, b_{i}(b)$ is linear, so it is trivial to prove $b_{*}=(\mu-(1-\alpha))^{-1}$ to be the unique solution of the fixed point equation $b_{i}(b)=b$. No matter how small $\sigma^{2}$ is, the fixed point equation $b_{i}(b)=b$ is instead a cubic, so that it has at most three real roots. By continuity, a positive solution $b_{\circ}$ must exist such that $\lim _{\sigma^{2} \rightarrow 0} b_{i}\left(b_{\circ}\right)=b_{\circ}=b_{*}$. The condition $\mu>1-\alpha$ is necessary and sufficient to have

$$
\lim _{\sigma^{2} \rightarrow 0} b_{i}^{\prime}\left(b_{*}\right)=\lim _{\sigma^{2} \rightarrow 0} b_{i}^{\prime}\left(b_{\circ}\right)=\frac{\mu}{1-\alpha}>1 .
$$

In such a case, since for any non-null $\sigma^{2}$ we have $\lim _{b \rightarrow+\infty} b_{i}(b)=0$, then by continuity at least another intersection of $b_{i}(b)$ with the bisector at a positive value $b_{+}$must exist too. Notice also that $b_{i}(0)<0$. This, jointly with the fact that for $\sigma^{2} \neq 0$ it is $\lim _{b \rightarrow-\infty} b_{i}(b)=0$, implies that by continuity at least an intersection of $b_{i}(b)$ with the bisector at a negative value $b_{-}$exists too. Therefore $\left(b_{-} b_{\circ}, b_{+}\right)$are the three solutions we were looking for.

Suppose now that $b_{-}$and $b_{+}$take finite values - that is $\left|b_{ \pm}\right| \leq M$ with $M$ being an arbitrarily large finite number - then $\lim _{\sigma^{2} \rightarrow 0} \sigma^{2}\left(1-b_{ \pm}\right)^{2}=0$ and so necessarily $b_{ \pm}=b_{\circ}>0$. Nevertheless $b_{-}<0$ so a first contradiction arises. Moreover if $b_{+}$is arbitrarily close to $b_{\circ}$ then by continuity $\lim _{\sigma^{2} \rightarrow 0} b_{i}^{\prime}\left(b_{+}\right)>1$ which, using the same argument as above, would imply the existence of a fourth root; a second contradiction arises. Hence we conclude $\left|b_{ \pm}\right|>M$ with $M$ arbitrarily large.

Finally, let me prove that, if and only if $\mu>1-\alpha$, a multiplicity arises for a $\sigma^{2}$ small enough. For $\mu<1-\alpha$, two cases are possible, either $0<\lim _{\sigma^{2} \rightarrow 0} b_{i}^{\prime}\left(b_{\circ}\right)<1$ or $\lim _{\sigma^{2} \rightarrow 0} b_{i}^{\prime}\left(b_{\circ}\right)<0$. The case $0<\lim _{\sigma^{2} \rightarrow 0} b_{i}^{\prime}\left(b_{\circ}\right)<1$ implies that for a sufficiently small $\sigma^{2}$ at least one intersection of $b_{i}(b)$ with the bisector must exist by continuity. Nevertheless, in this case, this intersection is unique as the existence of a second one would require $\max b_{i}^{\prime}(b)>1$ (given that $\lim _{b \rightarrow \pm \infty} b_{i}(b)=$ $\left.0_{ \pm}\right)$, but $\sup _{b} \lim _{\sigma^{2} \rightarrow 0} b_{i}^{\prime}(b)=\lim _{\sigma^{2} \rightarrow 0} b_{i}^{\prime}\left(b_{\circ}\right)$. With $\lim _{\sigma^{2} \rightarrow 0} b_{i}^{\prime}\left(b_{\circ}\right)<0$ instead - for a $\sigma^{2}$ small enough - the curve $b_{i}(b)$ is either strictly decreasing in the first quadrant $\left(b_{i}(b)>0, b>0\right)$ and never lies in the fourth quadrant $\left(b_{i}(b)<0, b<0\right)$, or is strictly decreasing in the fourth quadrant and never lies in the first quadrant. Hence, $\lim _{\sigma^{2} \rightarrow 0} b_{i}(b)$ can only have one intersection with the bisector.

\section{A.2 Proof. of proposition 2}

Taking the limit $\lim _{\sigma^{2} \rightarrow 0} \lim _{b \rightarrow b_{ \pm}}$(where $\left|b_{ \pm}\right|>M$ with $M$ arbitrarily large) to both sides of fixed point (17) with $b_{i}(b)=b$, we get

$$
\lim _{\sigma^{2} \rightarrow 0} \lim _{b \rightarrow b_{ \pm}}(1-\mu b)^{2} \sigma^{2}=-(1-\alpha)^{2}-\lim _{b \rightarrow b_{ \pm}} \frac{(1-\alpha)(1-\mu b)}{b}=(1-\alpha)(\mu-(1-\alpha)),
$$

which is positive provided $\mu>1-\alpha$. Then, given (16), we have (18).

The cross-sectional variance of individual expectations is given by $b^{2} \sigma^{2}$. Notice that

$$
\lim _{\sigma^{2} \rightarrow 0} \lim _{b \rightarrow b_{ \pm}}(1-\mu b)^{2} \sigma^{2}=\lim _{\sigma^{2} \rightarrow 0} \lim _{b \rightarrow b_{ \pm}} \mu^{2} b^{2} \sigma^{2}
$$


and hence,

$$
\lim _{\sigma^{2} \rightarrow 0} \lim _{b \rightarrow b_{ \pm}} b^{2} \sigma^{2}=\frac{(1-\alpha)(\mu-(1-\alpha))}{\mu^{2}}
$$

Given (14) it is easy to show that

$$
\lim _{\sigma^{2} \rightarrow 0} \lim _{b \rightarrow b_{ \pm}} \int \mathrm{E}\left[\theta \mid \mathrm{r}_{\mathrm{i}}\right] \mathrm{di}=-\lim _{\sigma^{2} \rightarrow 0} \lim _{b \rightarrow b_{ \pm}} \frac{(1-\alpha) b}{1-\mu b} \theta=\frac{1-\alpha}{\mu} \theta,
$$

so that the local price in the limit becomes $r_{i} \rightarrow 0$.

\section{A.3 Proof. of proposition 3}

We use a result from the proof above to establish

$$
\begin{aligned}
\lim _{\sigma^{2} \rightarrow 0} \lim _{b \rightarrow b_{ \pm}} \operatorname{var}_{i}\left(k_{i}\right) & =\lim _{\sigma^{2} \rightarrow 0} \lim _{b \rightarrow b_{ \pm}} \operatorname{var}_{i}\left(\frac{1-\mu b}{1-\alpha} \eta_{i}\right)= \\
& =\frac{\mu^{2}}{(1-\alpha)^{2}} \lim _{\sigma^{2} \rightarrow 0} \lim _{b \rightarrow b_{ \pm}} b^{2} \sigma^{2}=\frac{\mu-(1-\alpha)}{1-\alpha}>0 .
\end{aligned}
$$

Then $\lim _{\sigma^{2} \rightarrow 0} \lim _{b \rightarrow b_{\circ}} \operatorname{var}_{i}\left(k_{i}\right)=0$ follows as a consequence of $b_{\circ}$ being finite.

\section{A.4 Proof. of proposition 4}

The derivative of (17) with respect to $b$ is given by

$$
b_{i}^{\prime}(b)=-\frac{(1-\alpha) \mu\left(\mu^{2} b^{2} \sigma^{2}-2 \mu b \sigma^{2}-(1-\alpha)^{2}+\sigma^{2}\right)}{\left(\mu^{2} b^{2} \sigma^{2}-2 \mu b \sigma^{2}+(1-\alpha)^{2}+\sigma^{2}\right)^{2}} .
$$

For the perfect-information limit equilibrium, we have

$$
\lim _{\sigma^{2} \rightarrow 0} \lim _{b \rightarrow b_{\circ}} b_{i}^{\prime}(b)=\frac{\mu}{1-\alpha}>1 .
$$

For the dispersed-information limit equilibria notice that

$$
\lim _{\sigma^{2} \rightarrow 0} \lim _{b \rightarrow b_{ \pm}} b_{i}^{\prime}(b)=\frac{2(1-\alpha)-\mu}{\mu}
$$

after using (58) and (59) to compute the $\operatorname{limit}_{\lim _{\sigma^{2} \rightarrow 0}} \lim _{b \rightarrow b_{ \pm}} \mu^{2} b^{2} \sigma^{2}$. It is easy to prove that

$$
\lim _{\sigma^{2} \rightarrow 0} \lim _{b \rightarrow b_{ \pm}} b_{i}^{\prime}(b)<1 \text { and } \lim _{\sigma^{2} \rightarrow 0} \lim _{b \rightarrow b_{ \pm}} b_{i}^{\prime}(b)>-1,
$$

when $\mu>1-\alpha$ and $\alpha \in(0,1)$.

\section{A.5 Convergence under adaptive learning}

Consider now an infinite-horizon economy that consists of independent and identical realizations of the one-period economy described above. Following the approach of Marcet and Sargent (1989a,b) and Evans and Honkapohja (2001)), suppose agents behave like econometricians: they learn over time the right calibration of their forecasting rule. In particular, they individually set their weights in accordance with

$$
\begin{aligned}
b_{i, t} & =b_{i, t-1}+t^{-1} S_{i, t-1}^{-1} r_{i, t}\left(\theta_{t}-b_{i, t-1} r_{i, t}\right) \\
S_{i, t} & =S_{i, t-1}+(t+1)^{-1}\left(r_{i, t}^{2}-S_{i, t-1}\right),
\end{aligned}
$$


which is a recursive expression for a standard OLS regression, where the matrix $S_{i, t}$ is the estimated variance of the local price. Notice that, in contrast to the previous setting, here agents ignore the fact that learning is an ongoing collective process. In this sense this is a bounded rationality approach. The following formally defines adaptive stability.

Definition 4. A REE characterized by $\hat{b}$ is a locally learnable equilibrium if and only if a neighborhood $\digamma(\hat{b})$ of $\hat{b}$ exists such that, given an initial estimate $b_{i, 0} \in \digamma(\hat{b})$, it is $\lim _{t \rightarrow \infty} b_{i, t} \stackrel{a . s}{=}$. $\hat{b}$.

In other words, once estimates are close to the equilibrium values of a locally adaptively stable equilibrium, then convergence toward the equilibrium will almost surely occur. The result is stated by the proposition below.

Proposition 8. Whenever dispersed-information limit equilibria exist, they are locally adaptively stable, whereas the perfect-information limit equilibrium is not. The perfect-information limit equilibrium is locally adaptively stable when it is the unique limit equilibrium.

Proof. To check local learnability of the REE, suppose we are already close to the rest point of the system. That is, consider the case $\int \lim _{t \rightarrow \infty} b_{i, t} d i=\hat{b}$ where $\hat{b}$ is one among the equilibrium points $\left\{b_{-}, b_{\circ}, b_{+}\right\}$and so

$$
\lim _{t \rightarrow \infty} S_{i, t}=\operatorname{var}\left(r_{i, t}\right)=\frac{(1-\alpha)^{2}}{(1-\mu \hat{b})^{2}}+\sigma^{2},
$$

according to (15). From standard results in the stochastic approximation theory, we can write the associated ODE governing the stability around the equilibria as

$$
\begin{aligned}
\frac{\mathrm{d} b_{t}}{\mathrm{~d} t} & =\int \lim _{t \rightarrow \infty} \mathrm{E}\left[S_{i, t-1}^{-1} r_{i, t}\left(\theta_{t}-b_{i, t-1} r_{i, t}\right)\right] \mathrm{di}= \\
& =\operatorname{var}\left(r_{i, t}\right)^{-1} \int \mathrm{E}\left[r_{i, t}\left(\theta_{t}-b_{i, t-1} r_{i, t}\right)\right] \mathrm{di}= \\
& =\operatorname{var}\left(r_{i, t}\right)^{-1}\left(\operatorname{cov}\left(\theta, r_{i, t}\right)-b_{t} \operatorname{var}\left(r_{i, t}\right)\right)= \\
& =b_{i}\left(b_{t}\right)-b_{t},
\end{aligned}
$$

where we use relation (17).

The asymptotic behavior of statistical learning algorithms can be studied by stochastic approximation techniques. The proof uses well-known results to show that the asymptotic stability of the system around an equilibrium $\hat{b}$ is governed by the differential equation

$$
\frac{\mathrm{d} b}{\mathrm{~d} t}=b_{i}(b)-b,
$$

which is stable if and only if $b_{i}^{\prime}(\hat{b})<1$. This condition is known as the E-stability principle where $b_{i}(b)$ corresponds to the "projected T-map" in the adaptive learning literature. Therefore, adaptive stability implies eductive stability. Referring to Figure 3, the slope of the curves at the intersection with the bisector determines the stable or unstable nature of the equilibrium. It is straightforward to assess adaptive stability of dispersed-information limit equilibria: their existence relies precisely on the condition $b_{i}^{\prime}\left(b_{\circ}\right)>1$, which, since $b_{i}(b)$ is a cubic, necessarily implies $b_{i}^{\prime}\left(b_{ \pm}\right)<1$. 


\section{A.6 Decreasing returns to scale in intermediate production}

Consider the problem of intermediate producers in island $i$ being:

$$
\max _{Z_{(i)}}\left\{\mathrm{e}^{\theta+\eta_{i}} Z_{(i)}^{1-\vartheta} R_{i}-Z_{(i)} Q\right\}
$$

where $\vartheta \in(0,1)$ measures the returns to scale on intermediate production. The problem (61) generalizes (4)-(3), which obtain for $\vartheta=0$. The first order condition of (2) is

$$
(1-\vartheta) \mathrm{e}^{\theta+\eta_{i}} Z_{(i)}^{-\vartheta} R_{i}=Q,
$$

which can be expressed in log-deviations from the steady state as

$$
r_{i}=q+\vartheta z_{(i)}-\theta-\eta_{i}
$$

The latter combined with (9) and market clearing in the endowment market $\int z_{(i)} \mathrm{di}=0$ gives

$$
r_{i}=\mu \int \mathrm{E}\left[\theta \mid r_{i}\right] \mathrm{di}-\alpha \theta+\vartheta z_{(i)}-\eta_{i}
$$

Given that the quantity traded in equilibrium $k_{i}=\theta+\eta_{i}+(1-\vartheta) z_{(i)}$ is known by the final producer, then $r_{i}$ is observationally equivalent to

$$
\tilde{r}_{i}=r_{i}-\frac{\vartheta}{1-\vartheta} k_{i}=\mu \int \mathrm{E}\left[\theta \mid \tilde{r}_{i}\right] \mathrm{di}-\tilde{\alpha} \theta-\tilde{\eta}_{i},
$$

where

$$
\tilde{\alpha} \equiv \frac{(1-\vartheta) \alpha+\vartheta}{1-\vartheta}, \tilde{\eta}_{i} \equiv \frac{1}{1-\vartheta} \eta_{i}
$$

and $\mathrm{E}\left[\theta \mid r_{i}\right]=\mathrm{E}\left[\theta \mid \tilde{r}_{i}\right]$. Thus, the analysis in the text can be exactly replicated. A multiplicity arises in this case when $\mu>\tilde{\alpha}$, that is,

$$
\mu-\alpha>\frac{\vartheta}{1-\vartheta}
$$

which becomes increasingly restrictive as $\vartheta$ approaches 1 . At that point, the term $\tilde{\alpha}$, which measures the strength of the allocative effect, goes to infinity, so that $\theta$ is fully revealed irrespective of any finite value of $\mu$. Notice that for $\vartheta=1$ it is $k_{i}=\theta$, meaning that each producer knows the fundamental.

\section{B The Fragile Neutrality of Money}

\section{B.1 Proof. of proposition 5}

This proof. shows that, by fixing a profile of coefficients $\left(\mathrm{e}_{\theta}, \mathrm{e}_{\xi}, \mathrm{e}_{\eta}\right)$, a unique steady state and a unique log-normal deviation exist contingent on the stochastic state $\left(\theta,\left\{\eta_{i}, \xi_{i}\right\}_{i \in[0,1]}\right.$ for each variable in the model. 
First-order conditions and information acquisition in the extended model. For the sake of generality, I will focus on an extended version of the model that includes: the more general specification of the utility function (57) instead of (27), with convex disutility of labor measured by $\gamma$, and a more general specification of the intermediate technology,

$$
K_{i}^{s} \equiv e^{\eta_{i}} Z_{(i)}^{1-\zeta}
$$

instead of $(32)$, where $\zeta \in(0,1)$ features decreasing returns to scale in intermediate production. The baseline configuration obtains for $\gamma=\xi=0$. Let me start by providing the full list of first-order conditions and discuss how final producers' learning is affected in the extended model.

The household optimizes her problem when

$$
\begin{aligned}
Y_{i} & =P_{i}^{-\epsilon} P^{\epsilon} Y, \\
P & =e^{\theta} Y^{-\psi}, \\
W_{i} & =e^{\theta+\xi_{i}} L_{i}^{\gamma},
\end{aligned}
$$

where we already used (39) with $\delta / \bar{M}^{s}$ being normalized to one without any loss of generality.

Because of the convexity of the disutility of labor, wages cannot be determined independently from the quantity of labor hired. In this case, there is a conflict between observing the equilibrium wage at stage two and leaving working hours determined by actual demand in stage three. To circumvent this difficulty, we assume that, at the time when final producers make their decision $\left(K_{i}, P_{i}\right)$, they are informed about the labor supply schedule (foc3) posted by workers, i.e. final producers know the equilibrium wage contingent on each possible quantity traded. Given that producers on the same island share the same information, each of them will be able to forecast the quantity of labor hired (or expected to be hired) on a island. As a consequence, knowing the labor supply schedule is informationally equivalent to observing $e^{\theta+\xi_{i}}$, which is consistent with our baseline model.

The intermediate producers maximize their profits when

$$
R_{i}=(1-\zeta)^{-1} e^{-\eta_{i}} R Z_{(i)}^{\zeta} .
$$

Decreasing returns to scale on intermediate production makes $R_{i}$ depend on $Z_{(i)}$. At the same time $K_{i}=K_{i}^{s}=e^{\eta_{i}} Z_{(i)}^{1-\zeta}$ is known, as all producers on the same island have the same objectives and information. Hence, the information that final producers get from the local capital market is summarized by

$$
\hat{R}_{i} \equiv(1-\zeta) R_{i} K_{i}^{-\zeta /(1-\zeta)}=e^{-\eta_{i} /(1-\zeta)} R,
$$

which is identical to (40) up to a re-scaling of the variance of $\eta_{i}$. Therefore, final producers optimize their expected profits conditionally on $\left(\hat{R}_{i}, e^{\theta+\xi_{i}}\right)$, which constitutes the information transmitted by the prices of local inputs. The first-order conditions of final producers relative to the choices $\left(K_{i}, P_{i}\right)$ are

$$
\begin{aligned}
K_{i} & =\alpha^{1-\alpha}(1-\alpha)^{\alpha-1} R_{i}^{\alpha-1} \mathrm{E}\left[W_{i}^{1-\alpha} Y_{i} \mid \hat{R}_{i}, e^{\theta+\xi_{i}}\right], \\
P_{i} & =\epsilon(\epsilon-1)^{-1} \alpha^{-\alpha}(1-\alpha)^{\alpha-1} R_{i}^{\alpha} \mathrm{E}\left[W_{i}^{1-\alpha} \mid \hat{R}_{i}, e^{\theta+\xi_{i}}\right] .
\end{aligned}
$$

In the third stage, final producers hire working hours to satisfy actual demand. 
Log-normal algebra: preliminaries. This section aims to clarify some issues linked to the manipulation of log-linear relations. The requirement that equilibrium has a symmetric log-normal representation implies that the individual production $Y_{i}$ like any other variable in the model, has the form

$$
Y_{i}\left(\theta, \xi_{i}, \eta_{i}\right)=\bar{Y}_{i} e^{y_{i}\left(\theta, \xi_{i}, \eta_{i}\right)},
$$

where $y_{i} \equiv \log Y_{i}-\log \bar{Y}_{i}$ is defined as the distance of the logarithm of the particular realization $Y_{i}$ from its unconditional median $\bar{Y}_{i}$. In particular, the $\log$ deviation $y_{i}$ is a linear combination of the shocks

$$
y_{i}\left(\theta, \xi_{i}, \eta_{i}\right)=\mathrm{y}_{i, \theta} \theta+\mathrm{y}_{i, \xi} \xi_{i}+\mathrm{y}_{i, \eta} \eta_{i},
$$

where we use roman characters to index the relative weight of a shock in the combination. Notice that the stochastic steady state of individual production is given by its unconditional mean, $\bar{Y}_{i} e^{\operatorname{var}\left(y_{i}\right) / 2}$, with $\operatorname{var}\left(y_{i}\right)$ being the unconditional variance of $y_{i}$. By analogy, let us denote by $\mathrm{y}_{a, \theta}, \mathrm{y}_{a, \xi}$ and $\mathrm{y}_{a, \eta}$ the log-deviations of the aggregate $Y$ relative to a unitary increase in $\theta, \xi$ and $\eta$ respectively. Of course, by definition we have

$$
Y(\theta)=\bar{Y} e^{\mathrm{y}_{a, \theta} \theta},
$$

that is, $\mathrm{y}_{a, \xi}=\mathrm{y}_{a, \eta}=0$. At the same time, aggregate production can be obtained by aggregating (67) across agents

$$
\left(\int Y_{i}^{\frac{\theta-1}{\theta}} \mathrm{di}\right)^{\frac{\theta}{\theta-1}}=\bar{Y}_{i} e^{\mathrm{y}_{i, \theta} \theta+\frac{\theta-1}{2 \theta} \operatorname{var}\left(\mathrm{y}_{i, \xi} \xi_{i}+\mathrm{y}_{i, \eta} \eta_{i}\right)},
$$

so that, contrasting (67) and (70), we conclude $\mathrm{y}_{i, \theta}=\mathrm{y}_{a, \theta}$ for any $\theta>1$. In other words, the average $\log$ deviation of individual production is equal to the log deviation of aggregate production. This is true for whatever CES aggregation.

Therefore, in the following we are going to drop the distinction between individual and aggregate, focusing on $\left(\mathrm{y}_{\theta}, \mathrm{y}_{\xi}, \mathrm{y}_{\eta}\right)$ with the understanding that $\mathrm{y}_{\theta}=\mathrm{y}_{i, \theta}=\mathrm{y}_{a, \theta}, \mathrm{y}_{\xi}=\mathrm{y}_{i, \xi}$ and $\mathrm{y}_{\eta}=\mathrm{y}_{i, \eta}$. Regarding the deterministic component of (69) and (70), notice that

$$
\bar{Y}=\bar{Y}_{i} e^{\frac{\theta-1}{2 \theta} \operatorname{var}\left(\mathrm{y}_{i, \xi} \xi_{i}+\mathrm{y}_{i, \eta} \eta_{i}\right)},
$$

that is, both coincide only in the deterministic scenario, in which case they denote the deterministic steady state.

Uniqueness. Under the restriction of log linearity, the logarithm of each variable is determined by four components, which lie in the four independent complementary subspaces spanned by $\left(1, \theta, \xi_{i}, \eta_{i}\right)$. We can therefore study the reaction of the system to each component separately.

First, we consider the reaction to a unitary aggregate shock $\theta$. Let us determine the variables fixed at stage two for a given $\mathrm{e}_{\theta}$, which represents the weight on $\theta$ in the stochastic component of the individual expectation about $X=P Y^{\frac{1}{\epsilon}}$. Importantly, given that in the extended model $W_{i}$ moves with labor, and so with production, we need to keep track of the expected local wage. This corresponds to the following system of relations derived from (foc1), (foc 3 ),,(foc4),(foc5), 
(foc6), the market clearing condition $\int z_{(i)} \mathrm{di}=0$ and the final technology (30): ${ }^{23}$

$$
\begin{aligned}
\mathrm{y}_{\theta}^{e} & =\epsilon\left(\mathrm{e}_{\theta}-\mathrm{p}_{\theta}\right) \\
\mathrm{w}_{\theta}^{e} & =\gamma \mathrm{l}_{\theta}^{e}+1 \\
\mathrm{k}_{\theta}=0 & =(1-\alpha)\left(\mathrm{w}_{\theta}^{e}-\mathrm{r}_{\theta}\right)+\mathrm{y}_{\theta}^{e} \\
\mathrm{p}_{\theta} & =\alpha \mathrm{r}_{\theta}+(1-\alpha) \mathrm{w}_{\theta}^{e}, \\
(1-\alpha) \mathrm{l}_{\theta}^{e} & =\mathrm{y}_{\theta}^{e},
\end{aligned}
$$

where $\mathrm{w}_{\theta}^{e}, \mathrm{l}_{\theta}^{e}$ and $\mathrm{y}_{\theta}^{e}$ denote the impact of a unitary increase in $\theta$ on final producers' expectations about $W_{i}, L_{i}$ and $Y_{i}$ respectively. The expectation about $L_{i}$ is needed only with $\gamma \neq 0$ as the producers need to forecast the equilibrium wage to determine the optimal price. The system yields a unique solution

$$
\begin{aligned}
\mathrm{y}_{\theta}^{e} & =\Delta_{\theta}\left(\epsilon(1-\alpha)\left(\mathrm{e}_{\theta}-1\right)\right) \\
\mathrm{w}_{\theta}^{e} & =\Delta_{\theta}\left(\epsilon \gamma \mathrm{e}_{\theta}+(1-\alpha+\epsilon \alpha)\right) \\
\mathrm{r}_{\theta} & =\Delta_{\theta}\left(\epsilon(1+\gamma) \mathrm{e}_{\theta}-(\epsilon-1)(1-\alpha)\right) \\
\mathrm{p}_{\theta} & =\Delta_{\theta}\left(\epsilon(\alpha+\gamma) \mathrm{e}_{\theta}+1-\alpha\right), \\
\mathrm{l}_{\theta}^{e} & =\Delta_{\theta} \epsilon\left(\mathrm{e}_{\theta}-1\right)
\end{aligned}
$$

with $\Delta_{\theta} \equiv(1-\alpha+\epsilon(\alpha+\gamma))^{-1}$. Then, we can determine the equilibrium variables emerging at the third stage. The relations (foc2),(foc3), the final technology (30) and the definition of $X$ (44) determine the actual impact on $\mathrm{x}_{\theta}, \mathrm{y}_{\theta}, \mathrm{l}_{\theta}$ and $\mathrm{w}_{\theta}$ for a given $\mathrm{p}_{\theta}$. The corresponding system reads as

$$
\begin{aligned}
1-\psi_{\theta} & =\mathrm{p}_{\theta}, \\
\mathrm{w}_{\theta} & =\gamma \mathrm{l}_{\theta}+1 . \\
\mathrm{y}_{\theta} & =(1-\alpha) \mathrm{l}_{\theta}, \\
\mathrm{x}_{\theta} & =\epsilon^{-1} \mathrm{y}_{\theta}+\mathrm{p}_{\theta},
\end{aligned}
$$

In particular, note

$$
\mathrm{x}_{\theta}=\left(\left(\epsilon-\psi^{-1}\right)(\alpha+\gamma) \mathrm{e}_{\theta}+1-\alpha+\psi^{-1}(\alpha+\gamma)\right) \Delta_{\theta},
$$

which is consistent with (50). Moreover, for $\mathrm{e}_{\theta}=1$ we have $p_{\theta}=r_{\theta}=w_{\theta}=w_{\theta}^{e}=1$ and $y_{\theta}=y_{\theta}^{e}=l_{\theta}=l_{\theta}^{e}=0$, which entail the neutrality-of-money outcome.

Let us now turn our attention to the impact of idiosyncratic deviations, which only concern island-specific variables. Let us start with the variables fixed at the second stage. The system

\footnotetext{
${ }^{23}$ foc 2 together with the definition of $x$ can be used to recover the reaction of the individual expectation of the aggregate price and quantity in the final market. In this respect, it is important to stress that $y_{\theta}^{e}$ denotes the reaction of the individual expectation of individual demand, which is different from the reaction of the individual expectation of aggregate demand as agents cannot tell global and local fluctuations apart. For the same reason, $p_{\theta}$, which denotes the reaction of both the individual and the aggregate price, is different from the reaction of the individual expectation of the aggregate price.
} 
relative to $\xi$ and $\eta$ is determined by (foc1),(foc3),(foc4),(foc5),(foc6) and technologies (30) and (64), yielding:

$$
\begin{aligned}
\mathrm{y}_{\xi, \eta}^{e} & =\epsilon\left(\mathrm{e}_{\xi, \eta}-\mathrm{p}_{\xi, \eta}\right), \\
\mathrm{w}_{\xi, \eta}^{e} & =\gamma \mathrm{l}_{\xi, \eta}^{e}+1_{\xi} \\
\mathrm{r}_{\xi, \eta} & =\zeta \mathrm{z}_{\xi, \eta}-1_{\eta}, \\
(1-\zeta) \mathrm{z}_{\xi, \eta} & =\mathrm{k}_{\xi, \eta}-1_{\eta} \\
\mathrm{k}_{\xi, \eta} & =\mathrm{y}_{\xi, \eta}^{e}+(1-\alpha)\left(\mathrm{w}_{\xi, \eta}^{e}-\mathrm{r}_{\xi, \eta}\right) \\
\mathrm{p}_{\xi, \eta} & =\alpha \mathrm{r}_{\xi, \eta}+(1-\alpha) \mathrm{w}_{\xi, \eta}^{e} \\
(1-\alpha) l_{\xi, \eta}^{e} & =\mathrm{y}_{\xi, \eta}^{e}-\alpha \mathrm{k}_{\xi, \eta} .
\end{aligned}
$$

where I index the direct impact of the two shocks $\xi_{i}$ and $\eta_{i}$ on an individual expectation about $X$ with $1_{\xi}$ and $1_{\eta}$ respectively. There is a unique solution to the system given by

$$
\begin{aligned}
\mathrm{y}_{\xi}^{e} & =\Delta\left(\epsilon(\alpha \gamma-(1+\gamma) \alpha \zeta+1) \mathrm{e}_{\xi}-\epsilon(1-\alpha)\right) \\
\mathrm{r}_{\xi} & =\Delta\left(\epsilon \zeta(\gamma+1) \mathrm{e}_{\xi}-\zeta(1-\alpha)(\epsilon-1)\right) \\
\mathrm{w}_{\xi}^{e} & =\Delta\left(\gamma \epsilon \mathrm{e}_{\xi}+1+\zeta(\epsilon-1) \alpha\right) \\
\mathrm{p}_{\xi} & =\Delta\left(\epsilon(\gamma(1-\alpha)+\alpha \zeta(1+\gamma)) \mathrm{e}_{\xi}+1-\alpha\right) \\
\mathrm{l}_{\xi}^{e} & =\Delta\left(\epsilon \mathrm{e}_{\xi}-\alpha-\epsilon(1-\alpha)-\zeta \alpha(\epsilon-1)\right) \\
\mathrm{z}_{\xi}^{e} & =\Delta\left(\epsilon(\gamma+1) \mathrm{e}_{\xi}-(1-\alpha)(\epsilon-1)\right) \\
\mathrm{k}_{\xi} & =\Delta\left(\epsilon(\gamma+1)(1-\zeta) \mathrm{e}_{\xi}-(1-\alpha)(\epsilon-1)(1-\zeta)\right) .
\end{aligned}
$$

and

$$
\begin{aligned}
\mathrm{y}_{\eta}^{e} & =\Delta\left((\epsilon+\gamma \alpha \epsilon-\zeta(1+\gamma) \epsilon \alpha) \mathrm{e}_{\eta}+\alpha \epsilon(\gamma+1)\right) \\
\mathrm{r}_{\eta} & =\Delta\left(\epsilon \zeta(\gamma+1) \mathrm{e}_{\eta}-1-\gamma(\alpha+\epsilon(1-\alpha))\right) \\
\mathrm{w}_{\eta}^{e} & =\Delta\left(\gamma \epsilon \mathrm{e}_{\eta}+\alpha \gamma(\epsilon-1)\right) \\
\mathrm{p}_{\eta} & =\Delta\left(\epsilon(\gamma(1-\alpha)+\alpha \zeta(1+\gamma)) \mathrm{e}_{\eta}-\alpha(1+\gamma)\right) \\
\mathrm{l}_{\eta}^{e} & =\Delta\left(\epsilon \mathrm{e}_{\eta}+\alpha(\epsilon-1)\right) \\
\mathrm{z}_{\eta}^{e} & =\Delta\left(\epsilon(1+\gamma) \mathrm{e}_{\eta}+\alpha(1+\gamma)(\epsilon-1)\right) \\
\mathrm{k}_{\eta} & =\Delta\left(\epsilon(1+\gamma)(1-\zeta) \mathrm{e}_{\eta}+1+(\epsilon-1) \alpha+\gamma \epsilon\right) .
\end{aligned}
$$

with $\Delta \equiv(1+\gamma(\alpha(1-\epsilon)+\epsilon)+\zeta(\epsilon-1)(1+\gamma) \alpha)^{-1}$. The relations (foc1),(foc3) and (30) determine the actual cross-section of $y_{i}, l_{i}$ and $w_{i}$ as

$$
\begin{aligned}
\mathrm{y}_{\xi, \eta} & =-\epsilon \mathrm{p}_{\xi, \eta}, \\
\mathrm{w}_{\xi, \eta} & =\gamma \mathrm{l}_{\xi, \eta}+1_{\xi}, \\
\mathrm{y}_{\xi, \eta} & =\alpha \mathrm{k}_{\xi, \eta}+(1-\alpha) \mathrm{l}_{\xi, \eta},
\end{aligned}
$$

for given $\mathrm{p}_{\xi, \eta}$ and $\mathrm{k}_{\xi, \eta}$.

Finally, to find the deterministic steady state we can use seven relations (foc2), (foc3), (foc4), (foc5), (foc6), (30), (64), to generate a log-linear system in seven deterministic unknowns, namely $\log Y=\log Y_{i}, \log P=\log P_{i}, \log R_{i}, \log W_{i}, \log R, \log L=\log L_{i}, \log K=\log K_{i}=$ 
$Z^{1-\zeta}$ (implicitly, expectations about deterministic components are trivially correct), which has a unique solution. The unique stochastic steady state (expressed in log terms) is given by adding to the deterministic steady state of each variable (expressed in log terms) half of the variance of its stochastic component. As a result, in the stochastic steady state island-specific and aggregate variables differ for a second-order term.

\section{B.2 Proof. of proposition 6}

Given that all agents use the rule (54), then the average expectation is

$$
\int \mathrm{E}\left[x \mid w_{i}, r_{i}\right] \mathrm{di}=\frac{a+(1-\phi) b}{1-\phi b} \theta .
$$

Hence, an individual expectation can be rewritten as

$$
\begin{aligned}
& \mathrm{E}\left[x \mid w_{i}, r_{i}\right]=b\left(\phi \frac{a+(1-\phi) b}{1-\phi b} \theta+(1-\phi) \theta+\eta_{i}\right)+a\left(\theta+\xi_{i}\right), \\
& \mathrm{E}\left[x \mid w_{i}, r_{i}\right]=b\left(\frac{1-\phi+\phi a}{1-\phi b} \theta+\eta_{i}\right)+a\left(\theta+\xi_{i}\right) .
\end{aligned}
$$

The actual law of motion of the aggregate component of island-specific demand (50) is given by

$$
x=(1-\beta) \theta+\beta \frac{a+(1-\phi) b}{1-\phi b} \theta,
$$

which is a linear combination of exogenous shocks only. Now we can compute a fixed point equation working out the following orthogonal restrictions

$$
\begin{aligned}
\mathrm{E}\left[w_{i}\left(x-b_{i} r_{i}-a_{i} w_{i}\right)\right] & =0, \\
\mathrm{E}\left[r_{i}\left(x-b_{i} r_{i}-a_{i} w_{i}\right)\right] & =0 .
\end{aligned}
$$

The bidimensional individual best reaction function reads as

$$
\begin{gathered}
a_{i}(a, b)=\frac{1-\beta+\beta \frac{a+(1-\phi) b}{1-\phi b}-b \frac{1-\phi+\phi a}{1-\phi b}}{1+\sigma_{\xi}^{2}} \\
b_{i}(a, b)=\frac{(1-\beta) \frac{1-\phi b}{1-\phi+\phi a}+\beta\left(\frac{a+(1-\phi) b}{1-\phi+\phi a}\right)-a\left(\frac{1-\phi b}{1-\phi+\phi a}\right)}{1+\left(\frac{1-\phi b}{1-\phi+\phi a}\right)^{2} \sigma^{2}}
\end{gathered}
$$

provided $b \neq \phi$. From (75) we can recover the expression for the equilibrium $a=a_{i}(a, b)$, that is

$$
a=\frac{(1-\beta)(1-b)}{1-\beta+(1-b \phi) \sigma_{\xi}^{2}} .
$$

Notice that, with $\sigma_{\xi}^{2} \neq 0, a$ takes finite values for any $b$. Plugging the expression above at the numerator of (76), we obtain a fixed point equation in $b$ :

$$
b_{i}(b)=\frac{\frac{(\phi-\beta) \sigma_{\xi}^{2}-(1-\beta)}{(\phi-1) \sigma_{\xi}^{2}-(1-\beta)} b-\frac{(1-\beta) \sigma_{\xi}^{2}}{(\phi-1) \sigma_{\xi}^{2}-(1-\beta)}}{1+\left(\frac{1-\phi b}{1-\phi+\phi a}\right)^{2} \sigma^{2}}
$$


which is a cubic continuous function. With $\sigma^{2}=0, b_{i}(b)$ is linear, so it is trivial to prove $\left(a_{*}, b_{*}\right)=(0,1)$ to be the unique solution of the fixed point equation (78). No matter how small $\sigma^{2}$ is the fixed point equation (78) is instead a cubic which can be satisfied at most by three real roots. By continuity, a positive solution $b_{\circ}$ must exist such that $\lim _{\sigma^{2} \rightarrow 0} b_{i}\left(b_{\circ}\right)=b_{\circ}=b_{*}$. The condition

$$
\sigma_{\xi}^{2}>\frac{1-\beta}{\phi-1} \text { with } \phi>1
$$

is necessary and sufficient to have

$$
\lim _{\sigma^{2} \rightarrow 0} b_{i}^{\prime}\left(b_{*}\right)=\frac{(\phi-\beta) \sigma_{\xi}^{2}-(1-\beta)}{(\phi-1) \sigma_{\xi}^{2}-(1-\beta)}>1
$$

for any $\beta<1$. In such a case, given that

$$
\lim _{\sigma^{2} \rightarrow 0} b_{i}^{\prime}\left(b_{\circ}\right)=\lim _{\sigma^{2} \rightarrow 0} b_{i}^{\prime}\left(b_{*}\right)
$$

and that for $\sigma^{2} \neq 0$ we have $\lim _{b \rightarrow+\infty} b_{i}(b)=0$, then by continuity at least another intersection of $b_{i}(b)$ with the bisector at a positive value $b_{+}$must exist too. Moreover, (79) also implies $b_{i}(0)<0$. This, jointly with the fact that for $\sigma^{2} \neq 0$ it is $\lim _{b \rightarrow-\infty} b_{i}(b)=0$, implies that, by continuity, at least an intersection of $b_{i}(b)$ with the bisector at a negative value $b_{-}$also exists. Therefore, $\left(b_{-} b_{\circ}, b_{+}\right)$are the three solutions we were looking for.

Now suppose that $b_{-}$and $b_{+}$take finite values - that is $\left|b_{ \pm}\right| \leq M$ with $M$ being an arbitrarily large finite number - then, since $a$ is finite too, $\lim _{\sigma^{2} \rightarrow 0} \sigma^{2}\left(\left(1-\phi b_{ \pm}\right) /(1-\phi+\phi a)\right)^{2}=0$ and so necessarily $b_{ \pm}=b_{\circ}>0$. Nevertheless, $b_{-}<0$ so a first contradiction arises. Furthermore, if $b_{+}$ is arbitrarily close to $b_{\circ}$ then by continuity $\lim _{\sigma^{2} \rightarrow 0} b_{i}^{\prime}\left(b_{+}\right)>1$ that, using the same argument as above, would imply the existence of a fourth root; a second contradiction arises. Hence we can conclude $\left|b_{ \pm}\right|>M$ with $M$ arbitrarily large.

Finally let me prove that a multiplicity arises for a small enough $\sigma^{2}$ if and only if (79) holds. Out of these conditions two cases are possible, either $0<\lim _{\sigma^{2} \rightarrow 0} b_{i}^{\prime}\left(b_{\circ}\right)<1$ or $\lim _{\sigma^{2} \rightarrow 0} b_{i}^{\prime}\left(b_{\circ}\right)<$ 0 . The case $0<\lim _{\sigma^{2} \rightarrow 0} b_{i}^{\prime}\left(b_{\circ}\right)<1$ yields that, for a sufficiently small $\sigma^{2}$ at least one intersection of $b_{i}(b)$ with the bisector must exist by continuity. Nevertheless, in this case, such an intersection is unique as the existence of a second one would require $\sup _{b} \lim _{\sigma^{2} \rightarrow 0} b_{i}^{\prime}(b)>1$, given that $\lim _{b \rightarrow \pm \infty} b_{i}(b)=0_{ \pm}$but $\sup _{b} \lim _{\sigma^{2} \rightarrow 0} b_{i}^{\prime}(b)=\lim _{\sigma^{2} \rightarrow 0} b_{i}^{\prime}\left(b_{\circ}\right)$. With $\lim _{\sigma^{2} \rightarrow 0} b_{i}^{\prime}\left(b_{\circ}\right)<0$ instead - for a sufficiently small $\sigma^{2}$ - the curve $b_{i}(b)$ is either strictly decreasing in the first quadrant $\left(b_{i}(b)>0, b>0\right)$ and never lies in the fourth quadrant $\left(b_{i}(b)<0, b<0\right)$, or strictly decreasing in the fourth quadrant and never lies in the first quadrant. Hence, $\lim _{\sigma^{2} \rightarrow 0} b_{i}(b)$ can only have one intersection with the bisector.

Let us now find $\hat{\tau}$. From (77), we get

$$
\lim _{b \rightarrow \pm \infty} a=\frac{1-\beta}{\phi \sigma_{\xi}^{2}} .
$$

Using (78), we have

$$
\lim _{\sigma^{2} \rightarrow 0} \lim _{b \rightarrow b_{ \pm}}\left(\frac{1-\phi b}{1-\phi+\phi a}\right)^{2} \sigma^{2}=\frac{(\phi-\beta) \sigma_{\xi}^{2}-(1-\beta)}{(\phi-1) \sigma_{\xi}^{2}-(1-\beta)}-1=\frac{(1-\beta) \sigma_{\xi}^{2}}{(\phi-1) \sigma_{\xi}^{2}-(1-\beta)} .
$$


Using the relation above, we can write the total relative precision of private information about $\theta$ as

$$
\begin{aligned}
\lim _{\sigma^{2} \rightarrow 0} \lim _{b \rightarrow b_{ \pm}} \tau=\lim _{\sigma^{2} \rightarrow 0} \lim _{b \rightarrow b_{ \pm}}\left(\sigma_{\xi}^{-2}+\left(\frac{1-\phi b}{1-\phi+\phi a}\right)^{-2} \sigma^{-2}\right)= & \\
& =\sigma_{\xi}^{-2}+\frac{(\phi-1) \sigma_{\xi}^{2}-(1-\beta)}{(1-\beta) \sigma_{\xi}^{2}}=\frac{\phi-1}{1-\beta} .
\end{aligned}
$$

that is positive provided $\phi>1$.

\section{B.3 Proof. of proposition 7}

The cross-sectional variance of individual expectations is given by $a^{2} \sigma_{\xi}^{2}+b^{2} \sigma^{2}$. Note that

$$
\begin{array}{r}
\lim _{\sigma^{2} \rightarrow 0} \lim _{b \rightarrow b_{ \pm}}\left(\frac{1-\phi b}{1-\phi}+\phi a\right)^{2} \sigma^{2}(1-\phi+\phi a)^{2}=\lim _{\sigma^{2} \rightarrow 0} \lim _{b \rightarrow b_{ \pm}}(1-\phi b)^{2} \sigma^{2}=\lim _{\sigma^{2} \rightarrow 0} \lim _{b \rightarrow b_{ \pm}} \phi^{2} b^{2} \sigma^{2}= \\
=\frac{(1-\beta) \sigma_{\xi}^{2}}{(\phi-1) \sigma_{\xi}^{2}-(1-\beta)}\left(1-\phi+\phi \frac{1-\beta}{\phi \sigma_{\xi}^{2}}\right)^{2}=(1-\beta)\left(\phi-1-\frac{1-\beta}{\sigma_{\xi}^{2}}\right) .
\end{array}
$$

We also have

$$
\lim _{\sigma^{2} \rightarrow 0} \lim _{b \rightarrow b_{ \pm}} \frac{(1-\phi b)^{2} \sigma^{2}}{\phi^{2}}=\lim _{\sigma^{2} \rightarrow 0} \lim _{b \rightarrow b_{ \pm}} b^{2} \sigma^{2} .
$$

Using the relations above, we get

$$
\begin{aligned}
\lim _{\sigma^{2} \rightarrow 0} \lim _{b \rightarrow b_{ \pm}}\left(a^{2} \sigma_{\xi}^{2}+b^{2} \sigma^{2}\right) & = \\
=\frac{1-\beta}{\sigma_{\xi}^{2} \phi^{2}}\left((\phi-1) \sigma_{\xi}^{2}-(1-\beta)\right)+\frac{(1-\beta)^{2}}{\phi^{2} \sigma_{\xi}^{2}}= & =\frac{(1-\beta)(\phi-1)}{\phi^{2}}
\end{aligned}
$$

that is positive provided $\phi>1$.

\section{B.4 Extensions of the signal extraction problem}

Here, we reconsider the signal extraction problem solved by proposition 6 assuming a more general specification of the additional signal, which was provided, in the original model, by the local wage. Suppose agents forecast (50) while observing (48) and an additional signal: ${ }^{24}$

$$
\hat{s}_{i}=\theta+\vartheta_{e} \int \mathrm{E}\left[x \mid \hat{s}_{i}, r_{i}\right] \mathrm{di}+\hat{\varphi}+\hat{\xi}_{i}
$$

where $\hat{\varphi}$ is normally distributed shock orthogonal to the fundamental $\theta$, and $\vartheta_{e}$ is a constant.

Combining linearly ${ }^{25} \hat{s}_{i}$ and $r_{i}$ we can obtain:

$$
s_{i} \equiv\left(1-\phi-\frac{\phi}{\vartheta_{e}}\right)^{-1}\left(r_{i}-\frac{\phi}{\vartheta_{e}} \hat{s}_{i}\right)=\theta+\vartheta \eta_{i}+\varphi+\xi_{i},
$$

\footnotetext{
${ }^{24}$ The signal can be interpreted as a sufficient statistics produced by the existence of separate additional signals, some being purely private and others being purely public.

${ }^{25}$ Of course, the expectation operator in $r_{i}$ should be written conditional on the $\hat{s}_{i}$ instead of $w_{i}$.
} 
where $\varphi$ and $\xi_{i}$ are re-scaled realizations of $\hat{\varphi}$ and $\hat{\xi}_{i}$ respectively, and $\vartheta$ is a constant. In the case $\vartheta_{e}=0$, this transformation is not needed and we will consider (84) with $\vartheta=0$ instead, that is, $s_{i}=\hat{s}_{i}$. Let us denote by $\sigma_{\varphi}^{2}$ the variance of the common stochastic component $\varphi$ which measures the commonality of the information structure. This specification nests several cases:

i) $\sigma_{\varphi}^{2}=0, \vartheta=0$ is when $\hat{s}_{i}$ is the wage signal studied in proposition 6 ;

ii) $\sigma_{\varphi}^{2} \neq 0, \vartheta=0$ is when $\hat{s}_{i}$ is an exogenous private signal with common noise;

iii) $\sigma_{\varphi}^{2}=0, \vartheta \neq 0$ is when $\hat{s}_{i}$ is an endogenous private signal like $r_{i}$;

iv) $\sigma_{\varphi}^{2} \neq 0, \vartheta \neq 0$ is when $\hat{s}_{i}$ is an endogenous private signal with common noise.

An equilibrium is characterized by the two coefficients $(\hat{a}, \hat{b})$ that weight $\hat{s}_{i}$ and $r_{i}$ respectively, such that the individual expectation expressed as a linear combination of these two signals is a rational expectation. Then, for each couple $(\hat{a}, \hat{b})$ another couple $(a, b)$ must exist where: $b$ weights $r_{i}$, and $a$ weights $s_{i}$, such that

$$
\mathrm{E}\left[x \mid r_{i}, s_{i}\right]=b\left(\phi \int \mathrm{E}\left[x \mid r_{i}, s_{i}\right] \mathrm{di}+(1-\phi) \theta+\eta_{i}\right)+a\left(\theta+\vartheta \eta_{i}+\varphi+\xi_{i}\right)
$$

is still a rational expectation.

We will show here that, in all of the cases i)-iv), the conditions for the existence of dispersed limit equilibria - characterized as $b \rightarrow b_{ \pm}$where $b_{ \pm}^{2} \sigma^{2}=\kappa$ with $\kappa$ finite - are identical to the ones uncovered in proposition 6.

Given that all agents use the linear rule above, the average expectation is

$$
\int \mathrm{E}\left[x \mid r_{i}, s_{i}\right] \mathrm{di}=\frac{a+(1-\phi) b}{1-\phi b} \theta+\frac{a}{1-\phi b} \varphi .
$$

Hence, an individual expectation can be rewritten as

$$
\mathrm{E}\left[x \mid r_{i}, s_{i}\right]=b\left(\frac{1-\phi+\phi a}{1-\phi b} \theta+\frac{\phi a}{1-\phi b} \varphi+\eta_{i}\right)+a\left(\theta+\vartheta \eta_{i}+\varphi+\xi_{i}\right) .
$$

The actual law of motion of the aggregate component of island-specific demand (50) is given by

$$
x=(1-\beta) \theta+\beta\left(\frac{a+(1-\phi) b}{1-\phi b} \theta+\frac{a}{1-\phi b} \varphi\right),
$$

as functions of weights and exogenous shocks only. The fixed point equation is defined by

$$
\begin{aligned}
& \mathrm{E}\left[s_{i}\left(x-b r_{i}-a s_{i}\right)\right]=0, \\
& \mathrm{E}\left[r_{i}\left(x-b r_{i}-a s_{i}\right)\right]=0,
\end{aligned}
$$

that give

$$
\begin{gathered}
a=\frac{1-\beta+\beta\left(\frac{a+(1-\phi) b}{1-\phi b}+\frac{a}{1-\phi b} \sigma_{\varphi}^{2}\right)-b\left(\frac{1-\phi+\phi a}{1-\phi b}+\frac{\phi a}{1-\phi b} \sigma_{\varphi}^{2}+\vartheta \sigma^{2}\right)}{1+\vartheta^{2} \sigma^{2}+\sigma_{\varphi}^{2}+\sigma_{\xi}^{2}} \\
b=\frac{\frac{(1-\beta)(1-\phi b)}{1-\phi+\phi a}+\beta\left(\frac{a+(1-\phi) b}{1-\phi+\phi a}+\frac{\phi a^{2}}{(1-\phi+\phi a)^{2}} \sigma_{\varphi}^{2}\right)-a\left(\frac{1-\phi b}{1-\phi+\phi a}+\frac{\phi a(1-\phi b)}{(1-\phi+\phi a)^{2}} \sigma_{\varphi}^{2}+\left(\frac{1-\phi b}{1-\phi+\phi a}\right)^{2} \vartheta \sigma^{2}\right)}{1+\left(\frac{1-\phi b}{1-\phi+\phi a}\right)^{2} \sigma^{2}+\left(\frac{\phi a}{1-\phi+\phi a}\right)^{2} \sigma_{\varphi}^{2}}
\end{gathered}
$$


provided $b \neq \phi^{-1}$. It is easy to prove by substitution that for $\sigma^{2} \rightarrow 0,(a, b)=(0,1)$ is a solution.

Let me now prove that the conditions for the existence of dispersed-information limit equilibria are the same in this extended version. We can solve (88) for $a$ to get

$$
a=\frac{(1-\beta)(1-b)-b(1-b \phi) \sigma^{2} \vartheta}{(1-\beta)\left(1+\sigma_{\varphi}^{2}\right)+(1-b \phi)\left(\sigma_{\xi}^{2}+\sigma^{2} \vartheta^{2}\right)}
$$

Let us look now for the solution at the limit $\sigma^{2} \rightarrow 0$ such that $b \rightarrow b_{ \pm}$where $\lim _{\sigma^{2} \rightarrow 0, b \rightarrow b_{ \pm}} b^{2} \sigma^{2} \rightarrow$ $\kappa$ with $\kappa$ being finite (so that $b_{ \pm} \rightarrow \pm \infty$ ). In such a case, the equilibrium value of $a$ takes finite values for any $b$, provided $\sigma_{\xi}^{2} \neq 0$, in particular:

$$
\lim _{\sigma^{2} \rightarrow 0, b \rightarrow b_{ \pm}} a=\frac{1-\beta}{\phi \sigma_{\xi}^{2}}
$$

which is identical to the dispersed-information limit equilibrium value found in proposition 6 .

Taking the same limit $\sigma^{2} \rightarrow 0, b \rightarrow b_{ \pm}$on both the right-hand side and left-hand side of (89) we obtain:

$$
b_{ \pm}=\left(\frac{-\frac{(1-\beta) \phi}{1-\phi+\phi a}+\beta\left(\frac{1-\phi}{1-\phi+\phi a}\right)-a\left(-\frac{\phi}{1-\phi+\phi a}-\frac{a \phi^{2}}{(1-\phi+\phi a)^{2}} \sigma_{\varphi}^{2}\right)}{1+\left(\frac{\phi}{1-\phi+\phi a}\right)^{2} \kappa+\left(\frac{\phi a}{1-\phi+\phi a}\right)^{2} \sigma_{\varphi}^{2}}\right) b_{ \pm}
$$

which finally gives

$$
\lim _{\sigma^{2} \rightarrow 0, \rightarrow b_{ \pm}} b^{2} \sigma^{2} \rightarrow \kappa=(1-\beta) \frac{\phi-1-a \phi}{\phi^{2}}=(1-\beta) \frac{(\phi-1) \sigma_{\xi}^{2}-(1-\beta)}{\phi^{2} \sigma_{\xi}^{2}},
$$

which is the same as found in proof. B.3. The condition for the non-negativity of $\kappa$ is $\sigma_{\xi}^{2}>$ $(1-\beta)(\phi-1)^{-1}$; that is, equal to (55). Note in particular that the condition is independent of $\vartheta$ or $\sigma_{\varphi}^{2}$, i.e. whether or not the signal embeds the average expectation or a common noise term respectively.

\section{B.5 Out-of-equilibrium selection}

In this section, I investigate the stability properties of the equilibria. The following analysis extends that in section 2.4 to a case where agents forecast an endogenous state and exogenous information is also available.

Convergence in higher-order beliefs. Let us start by assessing the iterative process of higher-order beliefs. Suppose that it is common knowledge that the individual weights given to the signals $\left\{\left(a_{i}, b_{i}\right)\right\}_{0}^{1}$ lie in a neighborhood $\Im(\hat{a}, \hat{b})$ of the equilibrium characterized by $(\hat{a}, \hat{b})$. Common knowledge of $\left(a_{i, 0}, b_{i, 0}\right) \in \Im(\hat{a}, \hat{b})$ for each $i$ implies that the average weights $\left(a_{0}, b_{0}\right)$ belong to $\Im(\hat{a}, \hat{b})$. Call $B:(a, b) \rightarrow\left(a_{i}, b_{i}\right)$ the mapping that gives, for a given couple of average weights $(a, b)$, a couple of individual optimal weights $\left(a_{i}, b_{i}\right)$. Since $B(a, b)$ is common knowledge then $\left(a_{0}, b_{0}\right) \in \Im(\hat{a}, \hat{b})$ implies that a second-order belief is rationally justified for which $\left(a_{i, 1}, b_{i, 1}\right)=B\left(a_{0}, b_{0}\right)$ for each $i$, so that $\left(a_{i, 1}, b_{i, 1}\right) \in B(\Im(\hat{a}, \hat{b}))$ and as a consequence $\left(a_{1}, b_{1}\right) \in B(\Im(\hat{a}, \hat{b}))$. Iterating the argument, we have that $\left(a_{\nu}, b_{\nu}\right) \in B^{\nu}(\Im(\hat{a}, \hat{b}))$. 
Definition 4 A REE characterized by $(\hat{a}, \hat{b})$ is a locally unique rationalizable outcome if and only if

$$
\lim _{\nu \rightarrow \infty} B^{\nu}(\Im(\hat{a}, \hat{b}))=(\hat{a}, \hat{b}) .
$$

From an operational point of view, local uniqueness requires that

$$
\left\|J(B)_{(\hat{a}, \hat{b})}\right\|<1
$$

where $J(B)_{(\hat{a}, \hat{b})}$ is the Jacobian of map $B$ calculated at the equilibrium values $(\hat{a}, \hat{b})$. The following proposition states a result that holds for all the cases investigated in this section.

Proposition 9. Whenever dispersed information limit equilibria exist, they are locally unique rationalizable outcomes, whereas this is never the case for the perfect-information limit equilibrium.

Proof. To check convergence in higher order beliefs we need to build up the Jacobian computed around the equilibria using (75)-(76). It is

$$
\lim _{\sigma^{2} \rightarrow 0} \lim _{b \rightarrow b_{ \pm}} J=\left(\begin{array}{ll}
\frac{\partial a_{i}}{\partial a} & \frac{\partial a_{i}}{\partial b} \\
\frac{\partial b_{i}}{\partial a} & \frac{\partial b_{i}}{\partial b}
\end{array}\right),
$$

with

$$
\begin{aligned}
& J_{11}=\frac{\beta \frac{1}{1-\phi b}-\frac{\phi b}{1-\phi b}}{1+\sigma_{\xi}^{2}}, \\
& J_{12}=\frac{(\beta-1)(a \phi+1-\phi)}{\left(1+\sigma_{\xi}^{2}\right)(1-b \phi)^{2}}, \\
& J_{21}=\frac{-\frac{(1-\beta)(1-b \phi)}{(a \phi-\phi+1)^{2}}}{1+\left(\frac{1-\phi b}{1-\phi+\phi a}\right)^{2} \sigma^{2}}+\frac{2 \phi\left((1-\beta) \frac{1-\phi b}{1-\phi+\phi a}+\beta\left(\frac{a+(1-\phi) b}{1-\phi+\phi a}\right)-a\left(\frac{1-\phi b}{1-\phi+\phi a}\right)\right) \frac{(1-\phi b)^{2} \sigma^{2}}{(1-\phi+\phi a)^{3}}}{\left(1+\left(\frac{1-\phi b}{1-\phi+\phi a}\right)^{2} \sigma^{2}\right)^{2}}, \\
& J_{22}=\frac{\frac{\beta-\phi+a \phi}{1-\phi+\phi a}}{1+\left(\frac{1-\phi b}{1-\phi+\phi a}\right)^{2} \sigma^{2}}+\frac{2 \phi\left((1-\beta) \frac{1-\phi b}{1-\phi+\phi a}+\beta\left(\frac{a+(1-\phi) b}{1-\phi+\phi a}\right)-a\left(\frac{1-\phi b}{1-\phi+\phi a}\right)\right) \frac{(1-\phi b) \sigma^{2}}{(1-\phi+\phi a)^{2}}}{\left(1+\left(\frac{1-\phi b}{1-\phi+\phi a}\right)^{2} \sigma^{2}\right)^{2}} .
\end{aligned}
$$

The latter terms become

$$
\begin{aligned}
& J_{21}=\frac{-\frac{(1-\beta)(1-b \phi)}{(a \phi-\phi+1)^{2}}}{1+\left(\frac{1-\phi b}{1-\phi+\phi a}\right)^{2} \sigma^{2}}+\frac{2 \phi \frac{b(1-\phi b)^{2}}{(1-\phi+\phi a)^{3}} \sigma^{2}}{1+\left(\frac{1-\phi b}{1-\phi+\phi a}\right)^{2} \sigma^{2}}, \\
& J_{22}=\frac{\frac{\beta-\phi+a \phi}{1-\phi+\phi a}}{1+\left(\frac{1-\phi b}{1-\phi+\phi a}\right)^{2} \sigma^{2}}+\frac{2 \phi \frac{b(1-\phi b)}{(1-\phi+\phi a)^{2}} \sigma^{2}}{1+\left(\frac{1-\phi b}{1-\phi+\phi a}\right)^{2} \sigma^{2}} .
\end{aligned}
$$

after substituting for (76). Notice that

$$
\lim _{\sigma^{2} \rightarrow 0} \lim _{b \rightarrow b_{ \pm}} J_{21} J_{12}=0
$$


since $a$ is always finite, $J_{21}$ goes to infinity of order $b_{ \pm}$whereas $J_{12}$ goes to an infinitesimal of order $b_{ \pm}^{-2}$ (as remember $\lim _{\sigma^{2} \rightarrow 0} b_{ \pm} \sigma^{2}$ is a finite value). Therefore the eigenvalues of the Jacobian are

$$
\lim _{\sigma^{2} \rightarrow 0} \lim _{b \rightarrow b_{ \pm}} J_{11}=\frac{1}{1+\sigma_{\xi}^{2}} \in(0,1)
$$

and

$$
\lim _{\sigma^{2} \rightarrow 0} \lim _{b \rightarrow b_{ \pm}} J_{22}=1-2 \frac{\Gamma}{1+\Gamma} \in(-1,1)
$$

where (see also (82))

$$
\Gamma \equiv \lim _{\sigma^{2} \rightarrow 0} \lim _{b \rightarrow b_{ \pm}}\left(\frac{1-\phi b}{1-\phi+\phi a}\right)^{2} \sigma^{2}=\frac{(1-\beta) \sigma_{\xi}^{2}}{(\phi-1) \sigma_{\xi}^{2}-(1-\beta)}>0
$$

if and only if (79) holds. Therefore, whenever dispersed-information limit equilibria exist they are stable outcomes of a convergent process in higher-order beliefs.

At the perfect-information limit equilibrium instead the Jacobian is given by

$$
\lim _{\sigma^{2} \rightarrow 0} \lim _{b \rightarrow 1} J=\left(\begin{array}{cc}
\frac{\beta-\phi}{\left(1+\sigma_{\xi}^{2}\right)(1-\phi)} & -\frac{1-\beta}{\left(1+\sigma_{\xi}^{2}\right)(1-\phi)} \\
-\frac{1-\beta}{1-\phi} & \frac{\beta-\phi}{1-\phi}
\end{array}\right) .
$$

The product of its eigenvalues (the determinant of $J$ ) is

$$
\Delta(J)=\frac{1-2 \beta+\phi}{\left(1+\sigma_{\xi}^{2}\right)(\phi-1)},
$$

and their sum (the trace of $J$ ) is

$$
\operatorname{Tr}(J)=\frac{\left(2+\sigma_{\xi}^{2}\right)(\phi-\beta)}{\left(1+\sigma_{\xi}^{2}\right)(\phi-1)} .
$$

Provided $\operatorname{Tr}(J)^{2}>4 \Delta(J)$, the largest real eigenvalue is greater than one whenever

$$
\frac{1}{2} \operatorname{Tr}(J)+\frac{1}{2} \sqrt{\operatorname{Tr}(J)^{2}-4 \Delta(J)}>1
$$

that is, $\operatorname{Tr}(J)>1+\Delta(J)$, which requires $\sigma_{\xi}^{2}(1-\beta)>0$ given that $\phi>1$ (this also implies $\Delta(J)>0)$. Let us now prove $\operatorname{Tr}(J)^{2}>4 \Delta(J)$, that is

$$
\frac{\left(2+\sigma_{\xi}^{2}\right)^{2}(\phi-\beta)^{2}}{\left(1+\sigma_{\xi}^{2}\right)(\phi-1)}>4(1-2 \beta+\phi),
$$

which is equivalent to

$$
\begin{aligned}
(\beta-\phi)^{2} \sigma_{\xi}^{2}+ & \left(4(\beta-\phi)^{2}-4(\phi-1)(\phi-2 \beta+1)\right) \sigma_{\xi}^{2}+ \\
+ & \left(4(\beta-\phi)^{2}-4(\phi-1)(\phi-2 \beta+1)\right)>0
\end{aligned}
$$

which holds whenever $(\phi-1)(\phi-2 \beta+1)<0$ that is always true for $\beta<1$ and $\phi>1$. Hence, the perfect-information limit equilibrium entails a divergence in higher-order belief dynamics.

Finally, notice that $J_{22}$ alone characterizes the univariate case when fixing $a=0$, which obtains at the limit $\sigma_{\xi}^{2} \rightarrow \infty$. 
The intuition for the result is similar to that discussed for the unidimensional case in Section 2.4. The local instability of the perfect-information outcome relies on the high elasticity of price signals to expectation, which is maximal when close to the perfect-information scenario. This generates a circle of high complementarity between input demand and price signal, which pushes the signal toward steady state, as the aggregate supply of inputs is predetermined. At the point where the variance of the common component of the price signal is of the same order of magnitude as that of the idiosyncratic component, the price signal loses informativeness. Thus, the circle of high complementarity eventually dampens and the dynamics converge to a fixed point featuring a dispersed-information equilibrium.

Convergence with adaptive learning. Let us turn our attention now to adaptive learning. At the end of each period, producers see the realized demand, so they can eventually revise their beliefs in light of the realized forecast error. Following the approach described in Section 2.4, suppose that, at time $t$, agents set their weights $\chi_{i, t} \equiv\left[a_{i, t} b_{i, t}\right]^{\prime}$ in accordance with the bivariate recursive OLS expression:

$$
\begin{aligned}
\boldsymbol{\chi}_{i, t} & =\chi_{i, t-1}+t^{-1} S_{i, t-1}^{-1} \boldsymbol{\omega}_{i, t}\left(x_{t}-\boldsymbol{\chi}_{i, t-1}^{\prime} \boldsymbol{\omega}_{i, t}\right) \\
S_{i, t} & =S_{i, t-1}+(t+1)^{-1}\left(\boldsymbol{\omega}_{i, t} \boldsymbol{\omega}_{i, t}^{\prime}-S_{i, t-1}\right),
\end{aligned}
$$

with $\boldsymbol{\omega}_{i, t} \equiv\left[\begin{array}{ll}w_{i, t} & r_{i, t}\end{array}\right]^{\prime}$, where $S_{i, t}$ is the current estimate of the variance-covariance matrix of price signals. The following proposition formally defines adaptive stability.

Definition 5. A REE characterized by $(\hat{a}, \hat{b})$ is a locally learnable equilibrium if and only if a neighborhood $\digamma(\hat{a}, \hat{b})$ of $(\hat{a}, \hat{b})$ exists such that, given initial estimates $\left(a_{i, 0}, b_{i, 0}\right) \in \digamma(\hat{a}, \hat{b})$, it is $\lim _{t \rightarrow \infty}\left(a_{i, t}, b_{i, t}\right) \stackrel{a . s .}{=}(\hat{a}, \hat{b})$.

In other words, once estimates are close to the equilibrium values of a locally adaptively stable equilibrium, then convergence toward the equilibrium will almost surely occur.

Proposition 10. Whenever dispersed information limit equilibria exist, they are locally adaptively stable, whereas the perfect-information limit equilibrium never is.

Proof. To check local learnability of the REE, suppose we are already close to the rest point of the system. That is, consider the case $\int \lim _{t \rightarrow \infty} a_{i, t} \operatorname{di}=\hat{a}$ and $\int \lim _{t \rightarrow \infty} b_{i, t} \operatorname{di}=\hat{b}$ where $(\hat{a}, \hat{b})$ characterizes an equilibrium, so that

$$
\lim _{t \rightarrow \infty} S_{i, t}=\mathrm{S}=\left(\begin{array}{cc}
1+\sigma_{\xi}^{2} & \frac{1-\phi+a \phi}{1-b \phi} \\
\frac{1-\phi+a \phi}{1-b \phi} & \left(\frac{1-\phi+a \phi}{1-b \phi}\right)^{2}+\sigma^{2}
\end{array}\right) .
$$

From standard results in the stochastic approximation theory, we can write the associated ODE governing the stability around the equilibria as

$$
\begin{aligned}
\frac{\mathrm{d} \boldsymbol{\chi}_{i, t}}{\mathrm{~d} t} & =\int \lim _{t \rightarrow \infty} \mathrm{E}\left[S_{i, t-1}^{-1} \boldsymbol{\omega}_{i, t}^{\prime}\left(x_{t}-\boldsymbol{\omega}_{i, t}^{\prime} \boldsymbol{\chi}_{i, t-1}\right)\right] \mathrm{di}= \\
& =\mathrm{S}^{-1} \int \mathrm{E}\left[\boldsymbol{\omega}_{i, t}^{\prime}\left(x_{t}-\boldsymbol{\omega}_{i, t}^{\prime} \boldsymbol{\chi}_{i, t-1}\right)\right] \mathrm{di}= \\
& =\mathrm{S}^{-1}\left(\operatorname{cov}\left(x, \boldsymbol{\omega}_{i, t}\right)-\boldsymbol{\chi}_{i, t-1}=\right. \\
& =B\left(a_{i, t-1}, b_{i, t-1}\right)-\left[a_{i, t-1} b_{i, t-1}\right]^{\prime},
\end{aligned}
$$


where we use relation (75)-(76). The result directly follows from the proof of proposition 9.

As before, the proof uses well-known results to show that the asymptotic stability of the system around an equilibrium $(\hat{a}, \hat{b})$ obtains if and only if

$$
J(B)_{(\hat{a}, \hat{b})}<1,
$$

which is a bi-dimensional transposition of the E-stability principle mentioned previously. Therefore, also in this case, equilibria that are locally unique rationalizable outcomes are also adaptively stable. 


\section{References}

Altig, D., L. Christiano, M. Eichenbaum, And J. Linde (2011): "Firm-Specific Capital, Nominal Rigidities and the Business Cycle," Review of Economic Dynamics, 14, 225-247.

Amador, M. And P.-O. Weill (2010): "Learning from Prices: Public Communication and Welfare," Journal of Political Economy, 118, 866 - 907.

_ (2012): "Learning from Private and Public Observations of Others' Actions," Journal of Economic Theory, 147, 910-940.

Angeletos, G.-M. And J. LA'O (2008): "Dispersed Information over the Business Cycle: Optimal Fiscal and Monetary Policy," Columbia University and MIT, Mimeo.

(2010): "Noisy Business Cycles," in NBER Macroeconomics Annual 2009, Volume 24, National Bureau of Economic Research, Inc, NBER Chapters, 319-378.

Angeletos, G.-M., G. Lorenzoni, and A. Pavan (2010): "Beauty Contests and Irrational Exuberance: A Neoclassical Approach," NBER Working Papers 15883, National Bureau of Economic Research, Inc.

Angeletos, G.-M. and A. Pavan (2007): "Efficient Use of Information and Social Value of Information," Econometrica, 75, pp. 1103-1142.

Angeletos, G.-M. And I. Werning (2006): "Crises and Prices: Information Aggregation, Multiplicity, and Volatility," American Economic Review, 96, 1720-1736.

Benhabib, J., P. Wang, and Y. Wen (2015): "Sentiments and Aggregate Fluctuations," Econometrica, Forthcoming.

Bergemann, D., T. Heumann, and S. Morris (2015): "Information and Volatility," Journal of Economic Theory, forthcoming.

Bergemann, D. And S. Morris (2013): "Robust Predictions in Games With Incomplete Information," Econometrica, 81, 1251-1308.

Bernheim, B. D. (1984): "Rationalizable Strategic Behavior," Econometrica, 52, 1007-28.

Boivin, J., M. P. Giannoni, And I. Mihov (2009): "Sticky Prices and Monetary Policy: Evidence from Disaggregated US Data," American Economic Review, 99, 350-84.

Burdett, K. And K. L. Judd (1983): "Equilibrium Price Dispersion," Econometrica, 51, 955-69.

Calvo, G. A. (1983): "Staggered prices in a utility-maximizing framework," Journal of Monetary Economics, 12, 383-398.

Carlsson, H. And E. van Damme (1993): "Global Games and Equilibrium Selection," Econometrica, 61, 989-1018. 
Carvalho, C. and F. Nechio (2016): "Factor Specificity and Real Rigidities," Review of Economic Dynamics, 22, 208-222.

Chahrour, R. and G. Gaballo (2017): "Learning from prices: amplification and business fluctuations," Working Paper Series 2053, European Central Bank.

Christiano, L. J., M. Eichenbaum, and C. L. Evans (2005): "Nominal Rigidities and the Dynamic Effects of a Shock to Monetary Policy," Journal of Political Economy, 113, 1-45.

David, J. M., H. A. Hopenhayn, and V. Venkateswaran (2016): "Information, Misallocation, and Aggregate Productivity," The Quarterly Journal of Economics, 131, 943-1005.

Desgranges, G. And C. Rochon (2013): "Conformism and public news," Economic Theory, $52,1061-1090$.

Eden, B. (2013): "Price Dispersion And Demand Uncertainty: Evidence From Us Scanner Data," Vanderbilt University Department of Economics Working Papers 13-00015, Vanderbilt University.

Evans, G. W. And S. M. Honkapohja (2001): Learning and Expectations in Macroeconomics, Princeton University Press, The MIT Press.

Ganguli, J. V. And L. YANG (2009): "Complementarities, Multiplicity, and Supply Information," Journal of the European Economic Association, 7, 90-115.

Grossman, S. J. (1976): "On the Efficiency of Competitive Stock Markets Where Trades Have Diverse Information," Journal of Finance, 31, 573-85.

Guesnerie, R. (1992): "An Exploration of the Eductive Justifications of the RationalExpectations Hypothesis," American Economic Review, 82, 1254-78.

(2005): Assessing Rational Expectations 2: Eductive Stability in Economics, vol. 1 of MIT Press Books, The MIT Press.

HAYEK, F. A. (1945): "The Use of knowledge in society," American Economic Review, 35, 519-530.

Hellwig, C., A. Mukherji, And A. Tsyvinski (2006): "Self-Fulfilling Currency Crises: The Role of Interest Rates," American Economic Review, 96, 1769-1787.

Hellwig, C. and V. Venkateswaran (2014): "Dispersed Information, Sticky Prices and Monetary Business Cycles: A Hayekian Perspective," New York University, Mimeo.

Hellwig, M. F. (1980): "On the aggregation of information in competitive markets," Journal of Economic Theory, 22, 477-498.

Kaplan, G. And G. Menzio (2015): "The Morphology Of Price Dispersion," International Economic Review, Forthcoming. 
LuCAS, R. E. J. (1972): "Expectations and the neutrality of money," Journal of Economic Theory, 4, 103-124.

(1973): "Some International Evidence on Output-Inflation Tradeoffs," American Economic Review, 63, 326-34.

(1975): "An Equilibrium Model of the Business Cycle," Journal of Political Economy, $83,1113-44$.

Mackowiak, B. and M. Wiederholt (2009): "Optimal Sticky Prices under Rational Inattention," American Economic Review, 99, 769-803.

Manzano, C. And X. Vives (2011): "Public and private learning from prices, strategic substitutability and complementarity, and equilibrium multiplicity," Journal of Mathematical Economics, 47, 346-369.

Marcet, A. and T. J. Sargent (1989a): "Convergence of Least-Squares Learning in Environments with Hidden State Variables and Private Information," Journal of Political Economy, $97,1306-22$.

(1989b): "Convergence of least squares learning mechanisms in self-referential linear stochastic models," Journal of Economic Theory, 48, 337-368.

Morris, S. And H. S. Shin (1998): "Unique Equilibrium in a Model of Self-Fulfilling Currency Attacks," American Economic Review, 88, 587-97.

Pearce, D. G. (1984): "Rationalizable Strategic Behavior and the Problem of Perfection," Econometrica, 52, 1029-50.

Phelps, E. S. (1970): Introduction: The New Microeconomics in Employment and In- flation Theory, History of Economic Thought Books, in E.S. Phelps et al., Microeconomic Foundations of Employment and Inflation Theory, new york: norton ed.

ReIs, R. (2006): "Inattentive Producers," Review of Economic Studies, 73, 793-821.

Sheshinski, E. And Y. Weiss (1977): "Inflation and Costs of Price Adjustment," Review of Economic Studies, 44, 287-303.

Smets, F. And R. Wouters (2007): "Shocks and Frictions in US Business Cycles: A Bayesian DSGE Approach," American Economic Review, 97, 586-606.

VIVES, X. (2017): "Endogenous Public Information and Welfare in Market Games," The Review of Economic Studies, 84, 935.

Walker, T. B. and C. H. Whiteman (2007): "Multiple equilibria in a simple asset pricing model," Economics Letters, 97, 191-196.

Woodford, M. (2003): Interest and prices: Foundations of a theory of monetary policy, Princeton University Press.

(2005): "Firm-Specific Capital and the New Keynesian Phillips Curve," International Journal of Central Banking, 1. 